Research Article

\title{
Identification and Validation of the lncRNA BACE1-AS as Immune-Related Influencing Factor in Tumorigenesis following Pan-Carcinoma Analysis
}

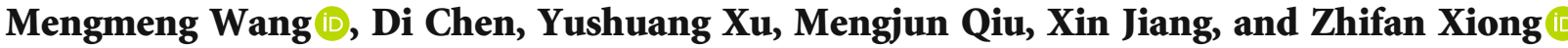 \\ Division of Gastroenterology, Liyuan Hospital, Tongji Medical College, Huazhong University of Science and Technology, \\ Wuhan 430077, China \\ Correspondence should be addressed to Zhifan Xiong; 1992ly0503@hust.edu.cn
}

Received 15 July 2021; Revised 9 October 2021; Accepted 2 November 2021; Published 8 December 2021

Academic Editor: Qiang Zhang

Copyright () 2021 Mengmeng Wang et al. This is an open access article distributed under the Creative Commons Attribution License, which permits unrestricted use, distribution, and reproduction in any medium, provided the original work is properly cited.

\begin{abstract}
Background. The lncRNA BACE1-AS was identified as a plasma molecular marker in the early diagnosis of Alzheimer's disease, but its role in tumors remains poorly defined. Methods. The expression patterns, genomic mutation, and prognostic significance of BACE1-AS in pan-cancers were compared by analyzing 32 types of tumors from The Cancer Genome Atlas and cBioPortal databases. The relationships between BACE1-AS expression levels and the degree of immune cell infiltration, immune components, and immune-related genes were explored. The possible molecular mechanisms of BACE1-AS in tumors were explored using gene set enrichment analysis (GSEA). Finally, the role of BACE1-AS in hepatocellular carcinoma was confirmed via quantitative real-time polymerase chain reaction (qRT-PCR). Results. BACE1-AS expression levels were significantly upregulated in LIHC, GBM, KIRC, CHOL, STAD, KICH, COAD, and PRAD. Higher expression levels of BACE1-AS were associated with worse overall survival in patients with HNSC and LIHC, while the opposite was found in PCPG and THCA. The overall mutation rate of BACE1-AS in pan-cancer was only approximately $0.9 \%$, and it occurred mainly in uveal melanoma and uterine carcinoma. Generally, BACE1-AS expression was negatively correlated with the immune microenvironment. BACE1-AS expression was mainly related to naïve B cells, activated memory CD4 T cells, monocytes, M1 macrophages, M2 macrophages, and resting mast cells. The potential mechanisms of BACE1-AS in tumors were mainly via regulating the activities of B cellmediated immunity, immune response regulating cell surface receptor signaling, RNA binding in posttranscriptional gene silencing, B cell receptor signaling pathways, and immune receptor activity. Finally, the qRT-PCR results confirmed that the expression levels of BACE1-AS in hepatocellular carcinoma cell lines were upregulated. Conclusions. Overall, our results suggest that BACE1-AS is associated with the expression, prognosis, and rate of immune cell infiltration of most tumors. Thus, BACE1AS may be a potential target for immunotherapies aimed at improving cancer patient outcomes.
\end{abstract}

\section{Introduction}

Malignant tumors are a major global public health problem that seriously threatens human health, and their overall incidence is rising worldwide [1]. In many developed countries, cancer-related deaths are second only to heart disease. In China, the incidence and mortality of cancer are the highest globally [2]. Worldwide, the top 10 cancers are lung, breast, stomach, colorectal, liver, esophagus, cervical, thyroid, pancreatic, and bladder. On average, more than 6,000 people die of cancer daily. In fact, approximately five people die of cancer every minute [3]. Thus, malignant tumors are a problem that threatens the health of all humans: they have a complex cause, their treatments have the worse therapeutic effects, and their prognosis is poor.

The theory that the genesis of a tumor is primarily determined by its genetic material is based on the tumor-centric view. Traditional methods such as surgical resection, radiotherapy, and chemotherapy are all derived from this theory; these methods all inevitably have the same destructive effect on normal tissues [4]. Recently, tumor immunotherapy targeting the immune system has revolutionized cancer treatment as 
well as given a new perspective in the mechanisms underlying tumor pathogenesis and drug resistance [5]. In particular, the current use of the cytotoxic T lymphocyte-associated protein 4 (CTLA4) and programmed cell death-1 (PD-1)/programmed cell death-ligand 1 (PD-L1) has become one of the most promising immunotherapies [6]. However, the efficacy of tumor immunotherapy is limited by the high degree of tumor microenvironment (TME) immunosuppression and the low immunogenicity of cancer cells [7].

Long noncoding RNAs (lncRNAs), a group of RNAs with a length of more than 200 nucleotides, do not contain protein-coding transcripts due to the lack of an open reading frame [8]. However, they play a significant role in tumor proliferation, invasion, metastasis, and chemoradiotherapy resistance [9]. The lncRNA MALAT1, by mediating the secretion of basic fibroblast growth factor (FGF-2), can inhibit inflammatory cytokine release and promote the proliferation, migration, and invasion of FTC133 cells [10]. Cao et al. found that the lncRNA MM2P modulates M2 macrophage polarization and weakens M2-mediated neovascularization [11]. BACE1-AS is transcribed from the opposite strand of $\beta$-secretase 1 (BACE1) and can pair with BACE1 mRNA to change its spatial structure and increase its stability to promote protein translation in a positive feed-forward fashion $[12,13]$. In other words, BACE1 and BACE1-AS can coregulate biological activities and processes and functionally interact with each other. BACE1 is a protease belonging to the $\beta$-secretase family and is thought to play an important role in Alzheimer's disease via the processing of amyloid precursor protein (APP) in neurotoxic $\mathrm{A} \beta$ peptides. Recently, BACE1 has been reported to be abnormally expressed in a variety of tumors and is associated with poor prognosis in colon cancer, non-small-cell lung cancer, invasive ductal carcinoma of the breast, glioblastoma, and gastric cancer [14-18].

Whether BACE1-AS has a similar effect in malignant tumors is unknown. Therefore, we conducted an in-depth analysis of 32 human tumors from The Cancer Genome Atlas (TCGA) database to further explore the role of BACE1-AS in tumors and its relationship with tumor immunity.

\section{Materials and Methods}

2.1. Pan-Cancer Data Source and Analysis. The expression matrix of BACE1-AS for 32 human tumors was obtained from TCGA database (https://portal.gdc.cancer.gov/), an open database covering 32 human cancer types, containing more than 30,000 tumor samples and information on the expression of more than 20,000 genes [19]. The mutations and copy number alterations (CNA) of BACE1-AS in pancancer were obtained by the cBioPortal for Cancer Genomics (http://www.cbioportal.org).

2.2. Kaplan-Meier Survival Analysis and Correlation between BACE1-AS Expression and Clinical Characteristics. The significance of BACE1-AS expression level for survival of 32 cancer types was achieved through $\mathrm{R}$ packages "survival" and "survminer" by Kaplan-Meier survival analysis. Overall survival (OS), disease-specific survival (DSS), progressionfree interval (PFI), and disease-free interval (DFI) were analyzed to evaluate prognostic value. The log-rank test was used to evaluate the statistical significance between them, and $P<0.05$ was considered statistically significant. The $\mathrm{R}$ "ggpubr" and "limma" packages were used to analyze the association between BACE1-AS expression and clinical characteristics including age, gender, and stage.

2.3. Mutation Analysis. Mutation data for 32 cancer types were downloaded by UCSC XENA (http://xena.ucsc.edu/), and tumor mutation load, the number of mutations per megabase, was calculated. The correlation between the expression of BACE1-AS and tumor mutation burden (TMB) or microsatellite instability (MSI) was calculated by the Spearman test. The results were represented by radar map drawn by the $\mathrm{R}$ "fmsb" package.

2.4. Correlation Analysis of BACE1-AS Expression and Tumor Microenvironment. The tumor microenvironment (TME), a special environment surrounding the presence of tumor cells, is composed of tumor stroma, adjacent cells, blood vessels, peripheral immune cells, and immune molecules [20]. The changes of these important components in the tumor microenvironment play a key role in tumor growth, invasion, metastasis, and immune tolerance. The $\mathrm{R}$ "estimation" package and "limma" package were used to analyze the association between BACE1-AS expression and tumor stromal or tumor immune cells. The higher the stromal scores and immune scores, the lower the purity of tumor cells.

2.5. Tumor-Infiltrating Immune Cell Analysis. The relative proportion of infiltrating immune cells in a pan-cancer patient was calculated by the CIBERSORT algorithm. The correlation between BACE1-AS expression and immune cells was analyzed by the Spearman correlation test. The $\mathrm{R}$ "ggpubr" package was used to analyze the correlation between 32 kinds of human tumors and 22 kinds of immune cells. The correlation between the expression of BACE1-AS and immune-related genes was performed by the R "reshape2" and the "RColorBrewer" packages.

2.6. Mechanism Analysis of BACE1-AS in Pan-Cancer. The potential mechanism of BACE1-AS in pan-cancer was mainly revealed by gene set enrichment analysis (GSEA) including Kyoto Encyclopedia of Genes and Genomes (KEGG) and Gene Ontology (GO) terms. The GSEA was performed by R "ggplot2," "enrichplot," “org.Hs.eg.db," and "clusterProfiler" packages based on "c2.cp.kegg.v7.1.symbols.gmt gene set" and "c5.all.v7.1.symbols.gmt". In order to obtain the normalized enrichment fraction, nominal (NOM) $P<0.05$ and false discovery rate $(\mathrm{FDR})<0.05$ were considered to be significantly enriched.

2.7. Validation of the Role of BACE1-AS in Hepatocellular Carcinoma. The prognostic value of BACE1-AS combined with coexpression immune-related checkpoint genes for hepatocellular carcinoma was analyzed, and a prognostic model was established by Cox regression analysis. Furthermore, the 
TABLE 1: Clinical features of pan-cancer from TCGA database.

\begin{tabular}{|c|c|c|c|c|c|c|}
\hline Abbreviation & Tumor type & Normal & Tumor & Female & Male & Average age \\
\hline ACC & Adrenocortical carcinoma & 0 & 79 & 48 & 31 & 46.70 \\
\hline BLCA & Bladder urothelial carcinoma & 19 & 408 & 114 & 313 & 68.17 \\
\hline BRCA & Breast invasive carcinoma & 113 & 1097 & 1197 & 13 & 58.21 \\
\hline CESC & Cervical squamous cell carcinoma and endocervical adenocarcinoma & 3 & 306 & 309 & 0 & 48.30 \\
\hline $\mathrm{CHOL}$ & Cholangiocarcinoma & 9 & 36 & 23 & 22 & 64.56 \\
\hline COAD & Colon adenocarcinoma & 35 & 461 & 234 & 262 & 67.51 \\
\hline DLBC & Lymphoid neoplasm diffuse large B cell lymphoma & 0 & 48 & 26 & 22 & 56.27 \\
\hline ESCA & Esophageal carcinoma & 11 & 162 & 29 & 144 & 63.82 \\
\hline GBM & Glioblastoma multiforme & 5 & 161 & 62 & 104 & 55.06 \\
\hline HNSC & Head and neck squamous cell carcinoma & 44 & 502 & 152 & 394 & 60.87 \\
\hline $\mathrm{KICH}$ & Kidney chromophobe & 24 & 65 & 38 & 51 & 52.60 \\
\hline KIRC & Kidney renal clear cell carcinoma & 72 & 531 & 217 & 386 & 61.54 \\
\hline KIRP & Kidney renal papillary cell carcinoma & 32 & 289 & 88 & 233 & 61.35 \\
\hline LAML & Acute myeloid leukemia & 0 & 151 & 68 & 83 & 50.00 \\
\hline LIHC & Liver hepatocellular carcinoma & 50 & 374 & 141 & 233 & 59.79 \\
\hline LUAD & Lung adenocarcinoma & 59 & 515 & 309 & 265 & 65.44 \\
\hline LUSC & Lung squamous cell carcinoma & 49 & 501 & 143 & 407 & 67.50 \\
\hline MESO & Mesothelioma & 0 & 86 & 15 & 71 & 62.95 \\
\hline $\mathrm{OV}$ & Ovarian serous cystadenocarcinoma & 0 & 379 & 379 & 0 & 60.16 \\
\hline PAAD & Pancreatic adenocarcinoma & 4 & 178 & 79 & 103 & 65.10 \\
\hline PCPG & Pheochromocytoma and paraganglioma & 3 & 183 & 103 & 83 & 47.32 \\
\hline PRAD & Prostate adenocarcinoma & 52 & 496 & 0 & 548 & 60.87 \\
\hline READ & Rectum adenocarcinoma & 10 & 166 & 84 & 92 & 64.19 \\
\hline SARC & Sarcoma & 2 & 263 & 145 & 121 & 61.12 \\
\hline SKCM & Skin cutaneous melanoma & 1 & 471 & 179 & 293 & 58.15 \\
\hline STAD & Stomach adenocarcinoma & 32 & 375 & 161 & 246 & 66.32 \\
\hline TGCT & Testicular germ cell tumors & 0 & 139 & 0 & 139 & 31.87 \\
\hline THCA & Thyroid carcinoma & 58 & 510 & 414 & 154 & 47.02 \\
\hline THYM & Thymoma & 2 & 119 & 60 & 61 & 57.91 \\
\hline UCEC & Uterine corpus endometrial carcinoma & 35 & 532 & 567 & 0 & 63.68 \\
\hline UCS & Uterine carcinosarcoma & 0 & 56 & 56 & 0 & 69.77 \\
\hline UVM & Uveal melanoma & 0 & 80 & 35 & 45 & 61.65 \\
\hline
\end{tabular}

expression level of BACE1-AS in HCC was detected by realtime quantitative reverse transcription PCR (qRT-PCR) at cell level. The sensitivity analysis of the common compounds was performed by R "pRRophetic" package.

2.8. Statistical Analysis. OS, DFI, DSS, and PFI were analyzed through $\mathrm{R}$ packages (R version 4.1.0) "survival" and "survminer" by the Kaplan-Meier method. The correlations between BACE1-AS expression level and TMB, MSI, stromal, tumor-infiltrating immune cells, and immune marker sets were calculated by the Spearman test. The correlation between BACE1-AS expression and clinical characteristics by the sensitivity analysis of the common compounds was analyzed by the Wilcoxon test. All statistics were achieved by SPSS statistical software (version 25.0.0). $P$ value $<0.05$ was considered statistically significant.

\section{Results}

3.1. BACE1-AS Is Abnormally Expressed in 32 Human Tumors. The pan-cancer data of 32 primary tumors are described in Table 1. Based on the analysis of 10,443 samples from TCGA database, we found that BACE1-AS expression levels were upregulated across most cancer types including LIHC, KIRP, KIRC, CHOL, STAD, KICH, COAD, and PRAD. In contrast, BACE1-AS expression levels in BRCA, UCEC, LUSC, and CESC were higher in normal samples than in tumors (Figure 1(a)). Further analysis was performed on tumors with more than 5 pairs of tumor and adjacent normal tissues. The results of paired difference analysis indicated that BACE1-AS expression levels were higher in LIHC, $\mathrm{CHOL}, \mathrm{KICH}$, and PRAD tumor tissues while downregulated in BRCA, UCEC, and LUSC (Figure 1(b)). 


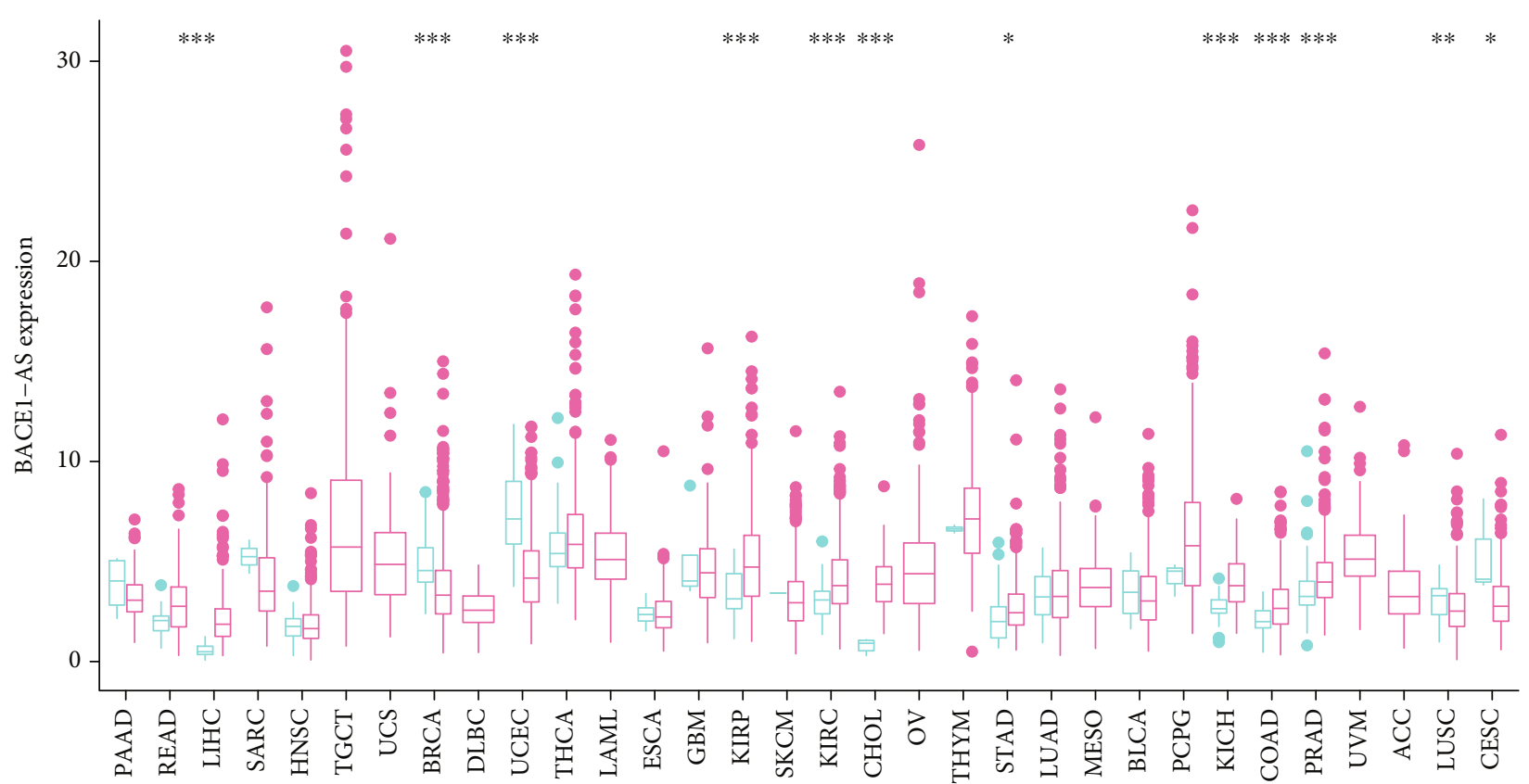

Type

白 Normal

追 Tumor

(a)
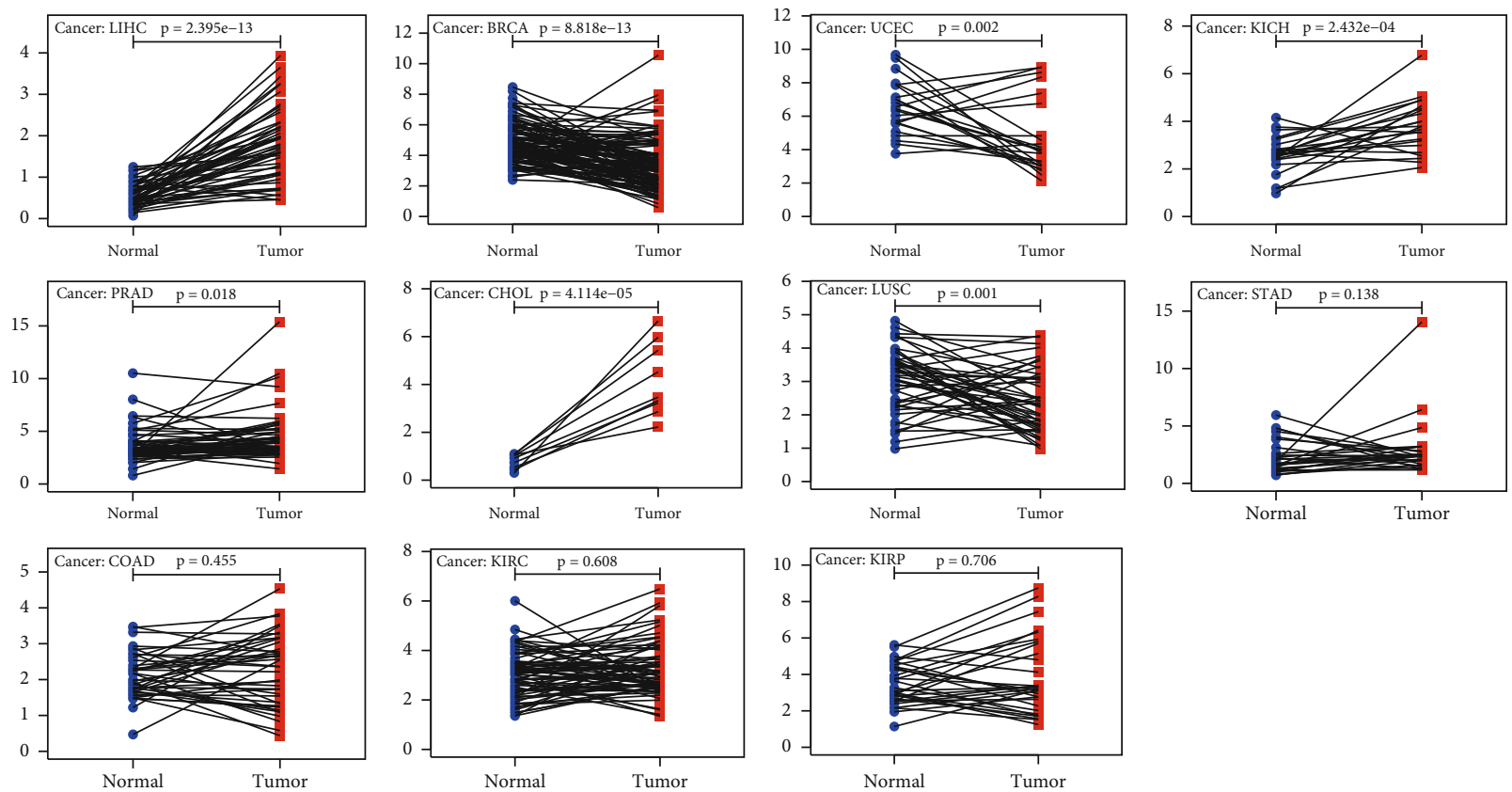

(b)

Figure 1: The abnormal expression level of BACE1-AS in 32 human tumors. (a) The expression level of BACE1-AS in pan-carcinomas from TCGA database. (b) Pairing differential analysis of BACE1-AS in tumors. ${ }^{*} P<0.05,{ }^{* *} P<0.01$, and ${ }^{* * *} P<0.001$.

3.2. The Prognostic Value of BACE1-AS in Pan-Cancer. The correlations between BACE1-AS expression levels and the prognosis of different cancers are depicted in Figure 2 and Supplementary Figure 1. Patients with downregulated BACE1-AS expression in ACC, COAD, KIRC, and LIHC had longer overall survival (OS), while the opposite was detected in PAAD and UVM patients (Figure 2(a)). The PFI analysis (Figure 2(b)) found that longer progressionfree intervals were associated with lower expression of BACE1-AS in ACC, LIHC, and PRAD. The DFI results 

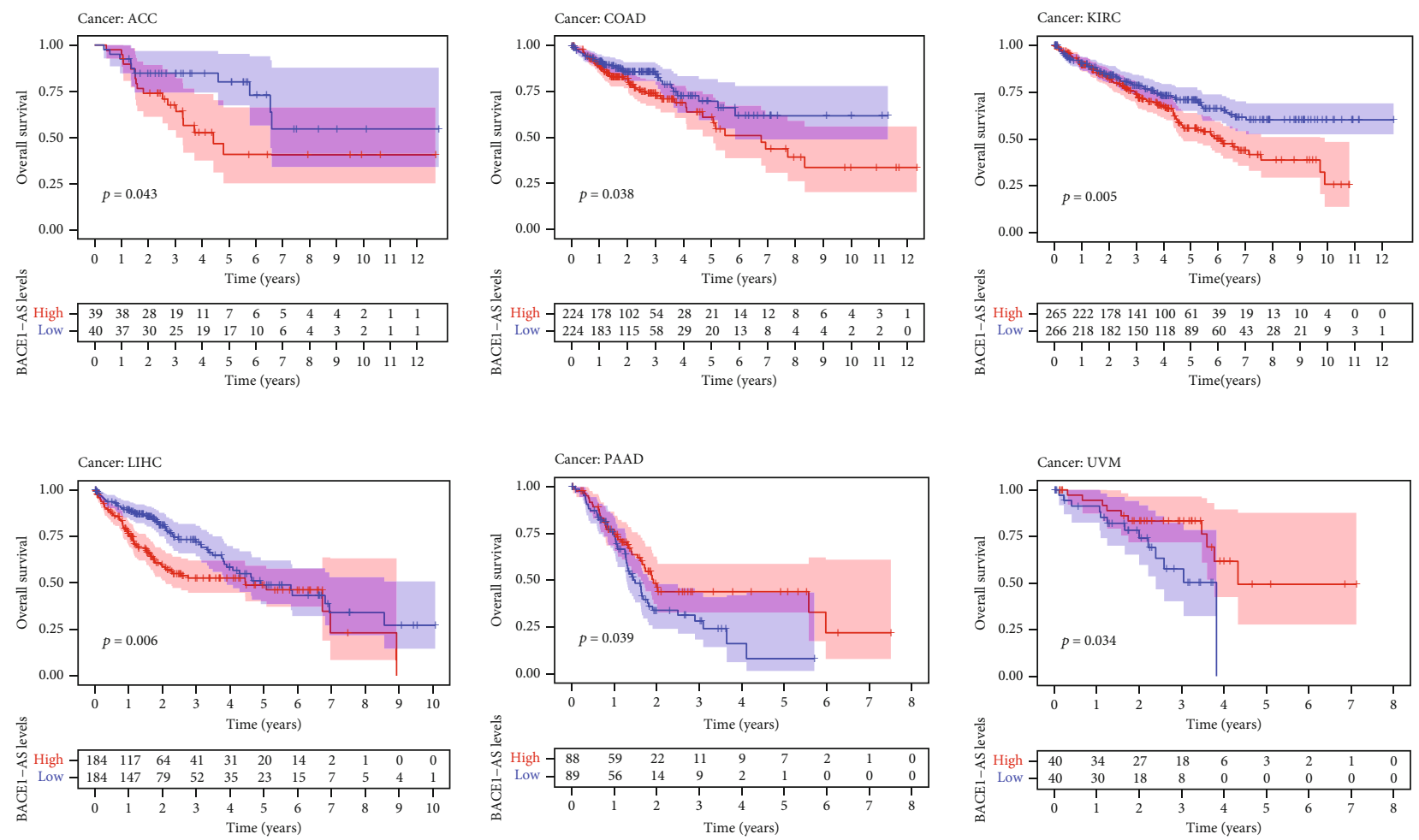

BACE1-AS levels

+ High

+ Low

(a)
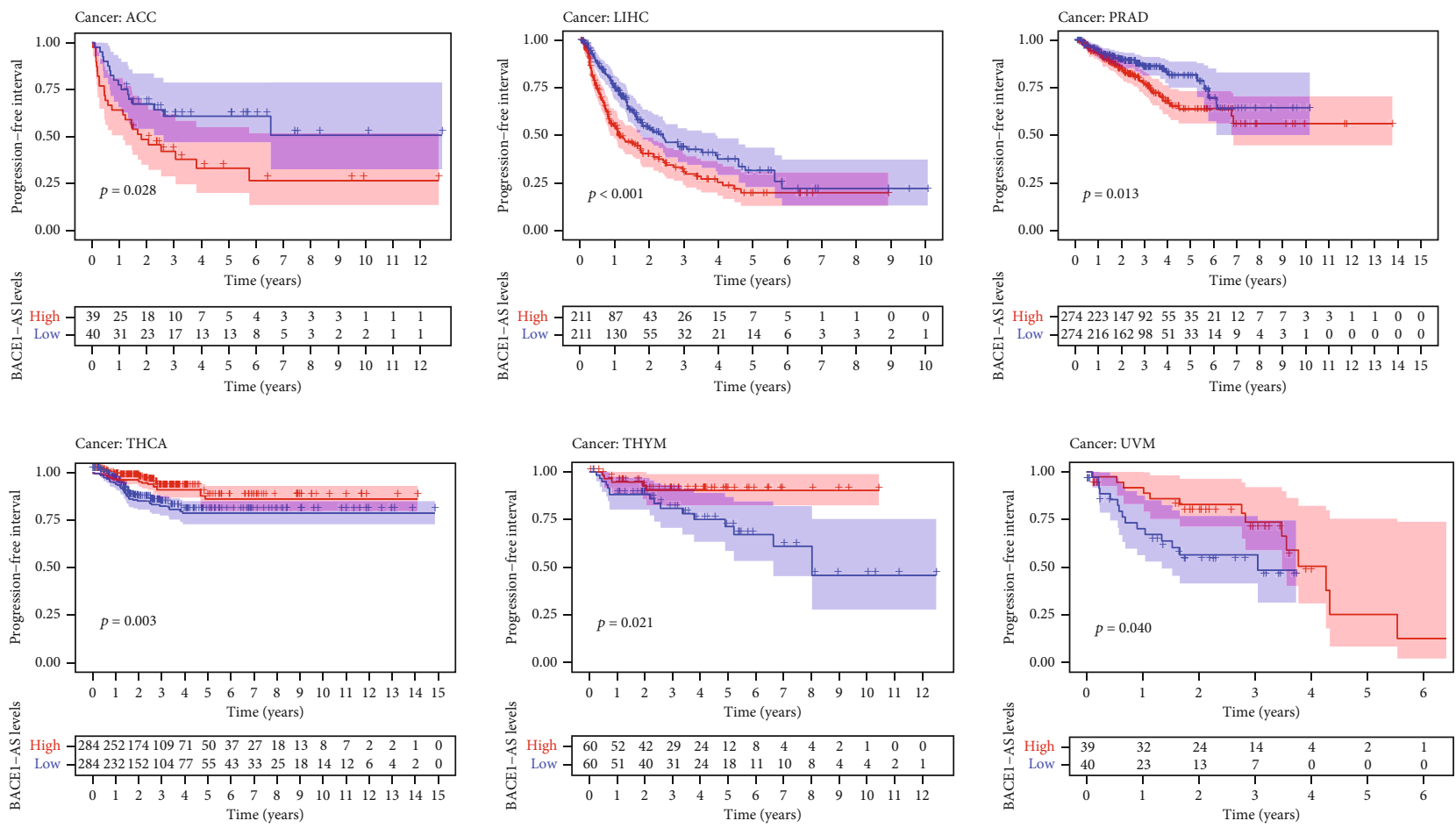

BACE1-AS levels

+ High

+ Low

(b)

Figure 2: Continued. 


$\begin{array}{lrr} & p \text { value } & \text { Hazard ratio } \\ \text { ACC } & 0.011 & 1.223(1.047-1.429) \\ \text { BLCA } & 0.206 & 0.943(0.862-1.033) \\ \text { BRCA } & 0.514 & 0.973(0.896-1.056) \\ \text { CESC } & 0.969 & 1.003(0.856-1.175) \\ \text { CHOL } & 0.644 & 0.937(0.712-1.233) \\ \text { COAD } & 0.106 & 1.118(0.977-1.279) \\ \text { DLBC } & 0.092 & 1.888(0.901-3.955) \\ \text { ESCA } & 0.520 & 1.080(0.855-1.363) \\ \text { GBM } & 0.677 & 0.985(0.918-1.057) \\ \text { HNSC } & 0.182 & 0.907(0.785-1.047) \\ \text { KICH } & 0.189 & 0.706(0.420-1.186) \\ \text { KIRC } & <0.001 & 1.204(1.116-1.299) \\ \text { KIRP } & 0.740 & 0.980(0.867-1.107) \\ \text { LAML } & 0.856 & 0.988(0.863-1.130) \\ \text { LIHC } & <0.001 & 1.201(1.086-1.328) \\ \text { LUAD } & 0.864 & 1.006(0.934-1.084) \\ \text { LUSC } & 0.391 & 0.955(0.860-1.061) \\ \text { MESO } & 0.676 & 0.969(0.836-1.123) \\ \text { OV } & 0.357 & 0.976(0.926-1.028) \\ \text { PAAD } & 0.022 & 0.803(0.664-0.969) \\ \text { PCPG } & 0.409 & 0.916(0.744-1.128) \\ \text { PRAD } & 0.640 & 0.910(0.614-1.350) \\ \text { READ } & 0.966 & 1.005(0.797-1.267) \\ \text { SARC } & 0.796 & 0.990(0.916-1.070) \\ \text { SKCM } & 0.429 & 0.965(0.884-1.054) \\ \text { STAD } & 0.139 & 0.907(0.796-1.032) \\ \text { TGCT } & 0.259 & 0.793(0.530-1.186) \\ \text { THCA } & 0.834 & 0.980(0.810-1.185) \\ \text { THYM } & 0.743 & 1.040(0.823-1.315) \\ \text { UCEC } & 0.982 & 0.999(0.906-1.101) \\ \text { UCS } & 0.342 & 0.956(0.872-1.049) \\ \text { UVM } & 0.222 & 0.863(0.682-1.093) \\ & & \end{array}$

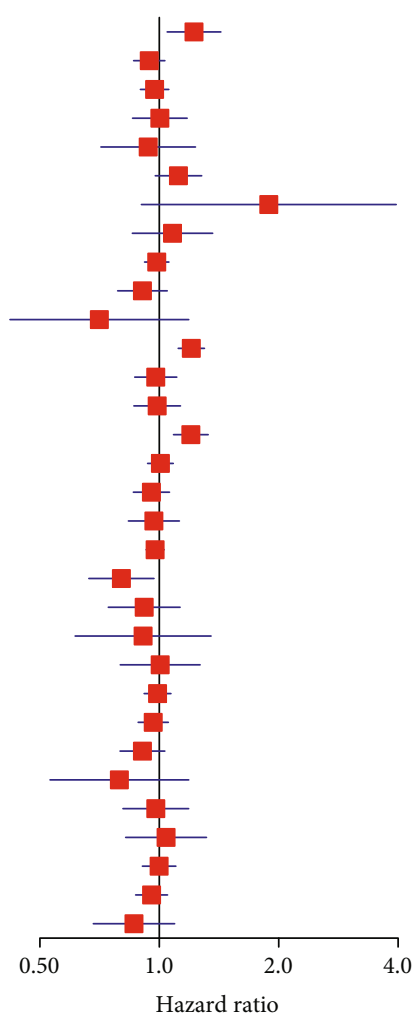

(c)

$\begin{array}{lr} & p \text { value } \\ \text { ACC } & 0.025 \\ \text { BLCA } & 0.562 \\ \text { BRCA } & 0.691 \\ \text { CESC } & 0.489 \\ \text { CHOL } & 0.699 \\ \text { COAD } & 0.405 \\ \text { DLBC } & 0.083 \\ \text { ESCA } & 0.633 \\ \text { GBM } & 0.229 \\ \text { HNSC } & 0.884 \\ \text { KICH } & 0.210 \\ \text { KIRC } & 0.015 \\ \text { KIRP } & 0.228 \\ \text { LIHC } & <0.001 \\ \text { LUAD } & 0.497 \\ \text { LUSC } & 0.443 \\ \text { MESO } & 0.555 \\ \text { OV } & 0.779 \\ \text { PAAD } & 0.001 \\ \text { PCPG } & 0.632 \\ \text { PRAD } & 0.160 \\ \text { READ } & 0.883 \\ \text { SARC } & 0.351 \\ \text { SKCM } & 0.534 \\ \text { STAD } & 0.577 \\ \text { TGCT } & 0.640 \\ \text { THCA } & 0.689 \\ \text { THYM } & 0.065 \\ \text { UCEC } & 0.318 \\ \text { UCS } & 0.325 \\ \text { UVM } & 0.412\end{array}$

Hazard ratio
$1.170(1.019-1.342)$
$0.975(0.893-1.063)$
$0.984(0.909-1.065)$
$1.052(0.911-1.214)$
$0.960(0.779-1.182)$
$1.056(0.929-1.200)$
$1.758(0.929-3.329)$
$1.052(0.854-1.296)$
$0.949(0.871-1.034)$
$0.990(0.865-1.133)$
$0.778(0.526-1.151)$
$1.103(1.019-1.193)$
$0.933(0.834-1.044)$
$1.150(1.060-1.248)$
$0.976(0.909-1.047)$
$0.954(0.847-1.075)$
$1.044(0.904-1.207)$
$1.006(0.962-1.053)$
$0.738(0.611-0.890)$
$0.972(0.865-1.092)$
$1.077(0.971-1.195)$
$1.015(0.833-1.236)$
$0.966(0.900-1.038)$
$0.977(0.909-1.051)$
$0.965(0.852-1.093)$
$0.986(0.929-1.046)$
$0.979(0.884-1.085)$
$0.841(0.700-1.011)$
$0.957(0.878-1.043)$
$0.951(0.862-1.051)$
$0.921(0.756-1.121)$

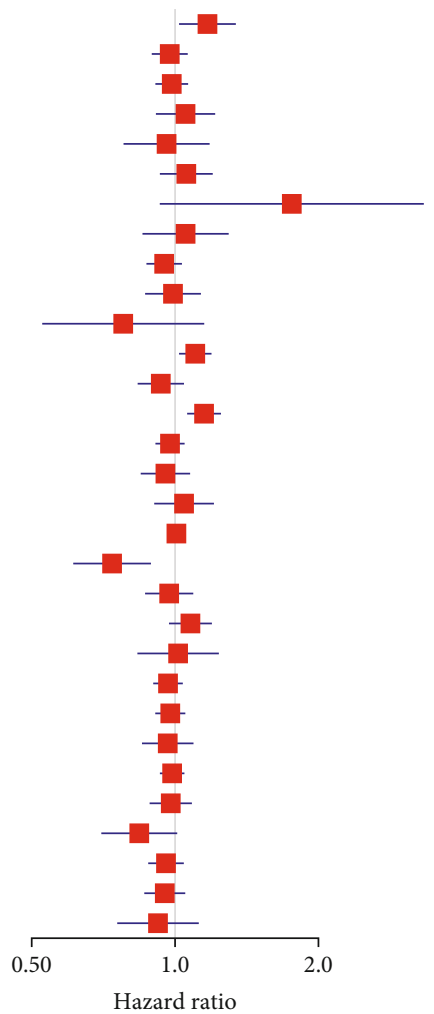

(d)

Figure 2: Prognostic value of BACE1-AS in different tumors. ( $a, b)$ Kaplan-Meier curves estimate the OS and PFI differences in pan-cancer. $(c, d)$ OS and PFI differences among pan-cancer analyzed by univariate regression analysis. Only survival curves with significant differences $(P<0.05)$ were shown. 

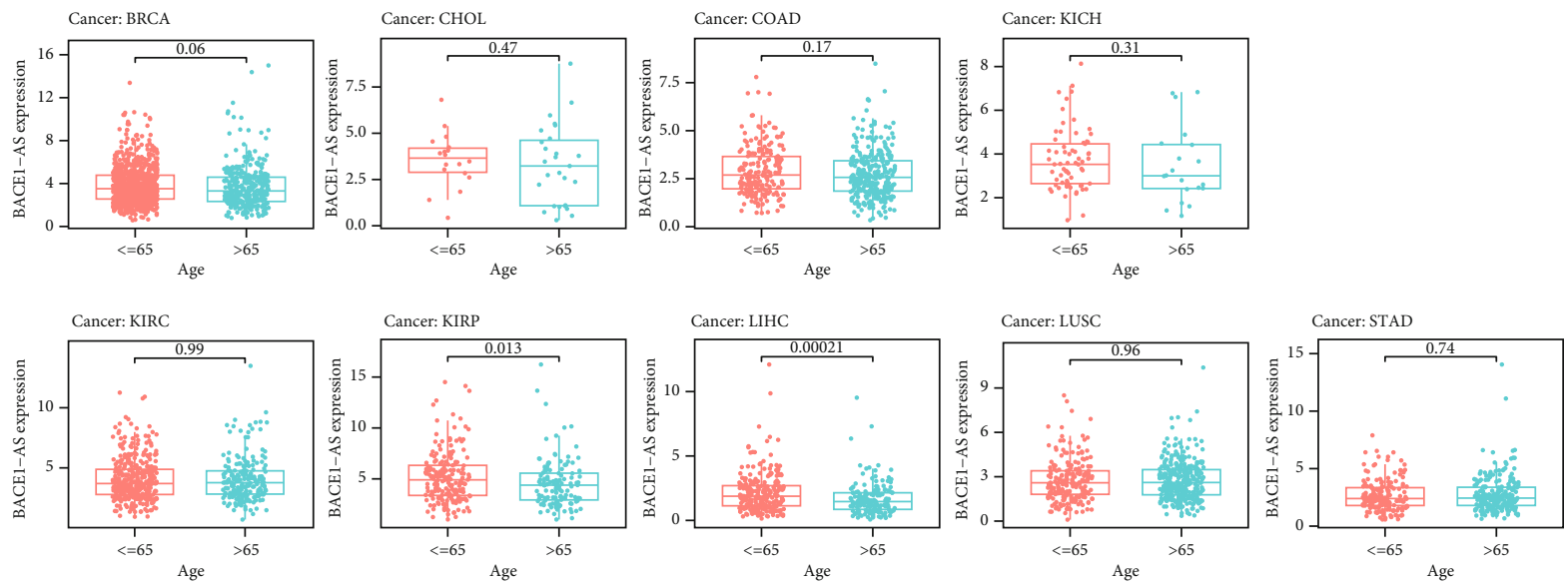

Age $<$ 俩<65

店 $>65$

(a)
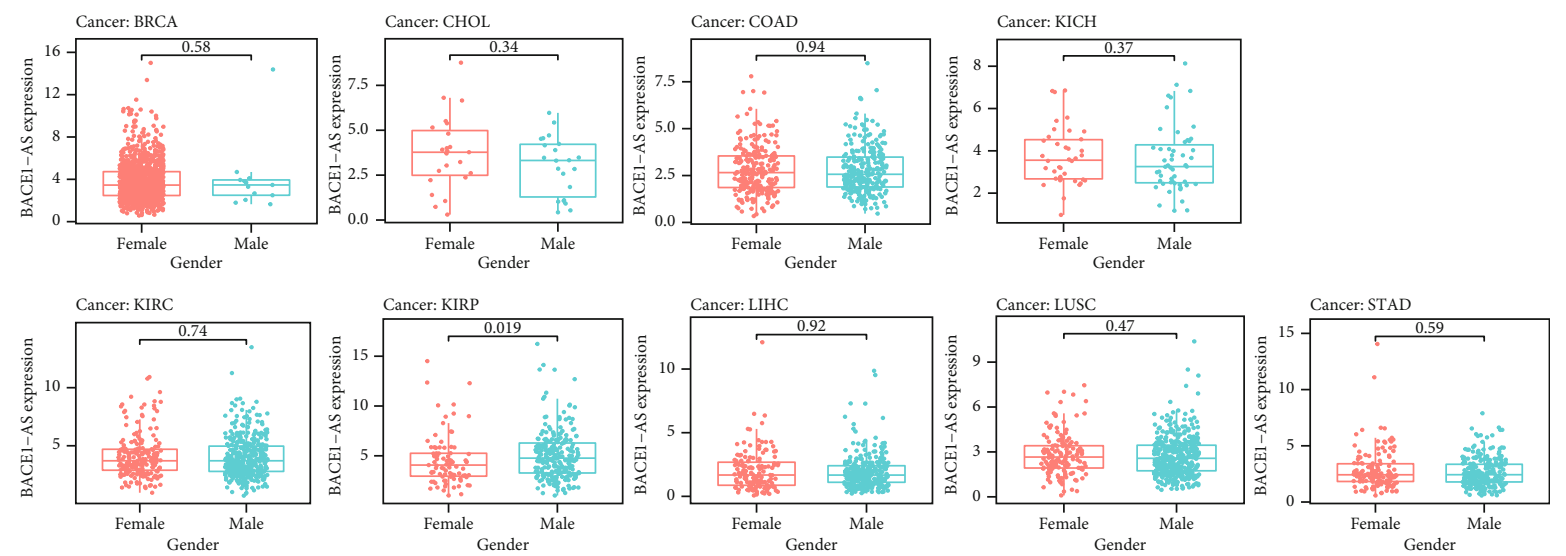

Gender

审 Female

它 Male

(b)
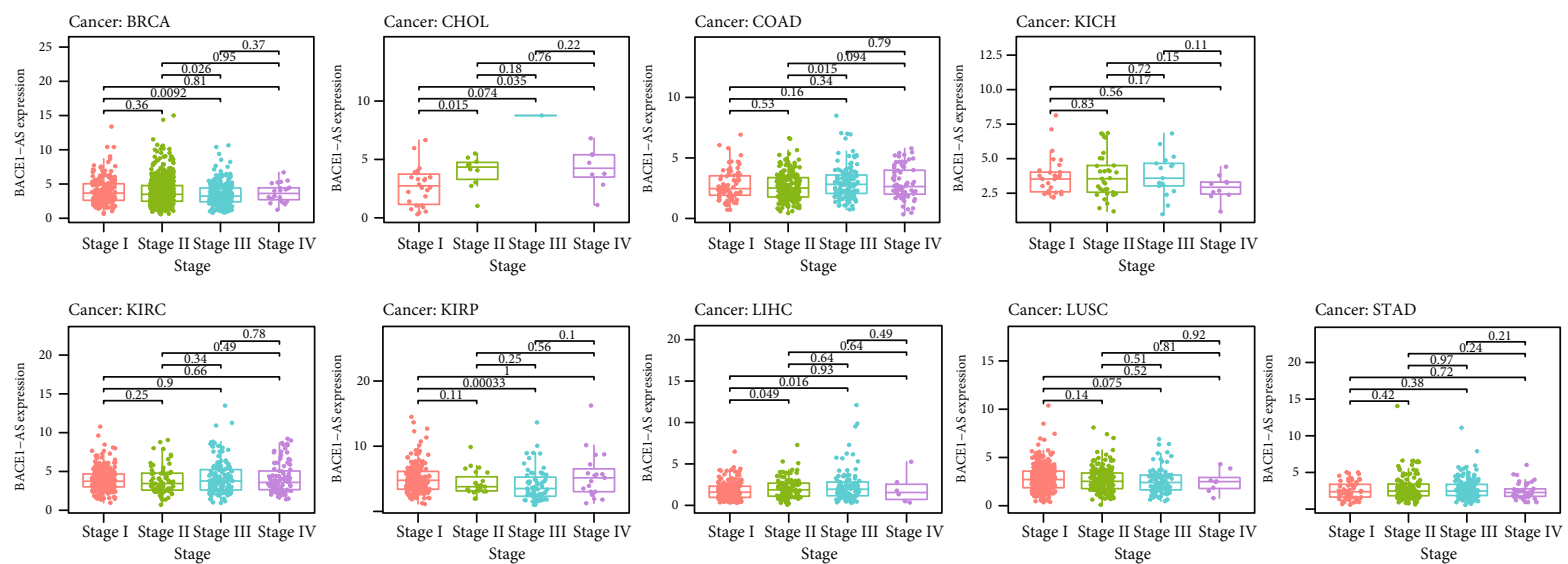

Stage

Stage I Stage III

Stage II

(c)

FIgURE 3: Correlation between the expression level of BACE1-AS and clinical characteristics. (a) Relationship between expression level of BACE1-AS and age. (b) Correlation analysis between expression level of BACE1-AS and gender. (c) The expression level of BACE1-AS in different clinical stages among different tumors. 


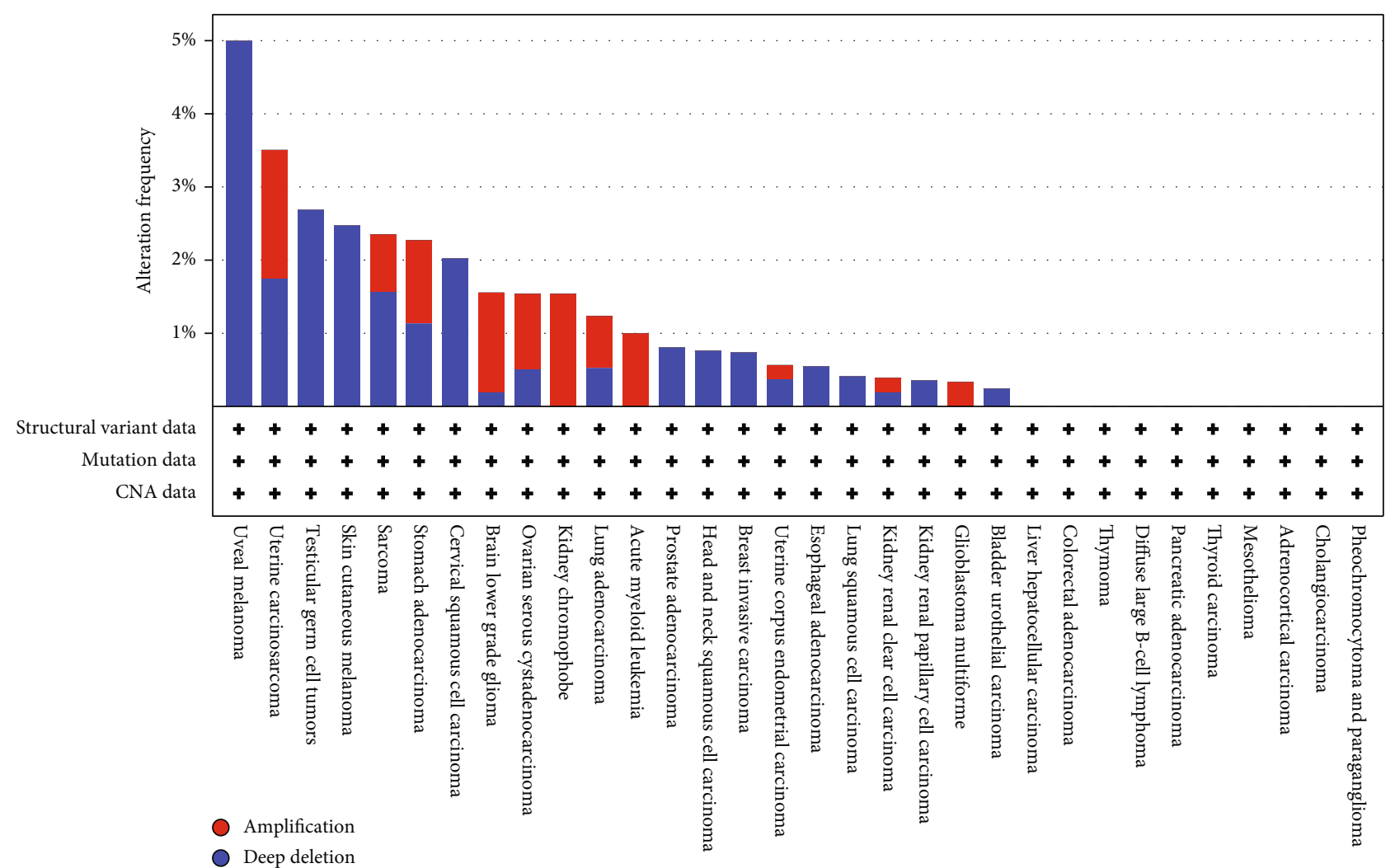

(a)

Mutation spectrum

Profiled for copy number alterations

TCGA PanCanAtlas cancer type acronym

Profiled for mutations

Profiled for structural variants

In PanCan pathway analysis

MSIsensor score

MSI MANTIS score

Overall survival (months)

Overall survival status

BACE1-AS

Genetic alteration

Mutation spectrum

Profiled for copy number alteration

TCGA PanCanAtlas cancer type

Profiled for structural variants

In PanCan pathway analysis

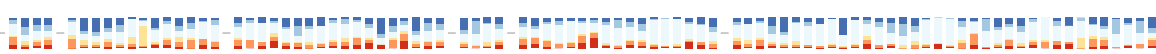
|

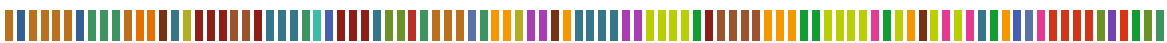

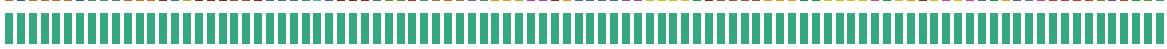
|

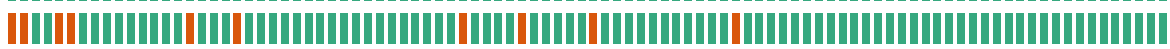

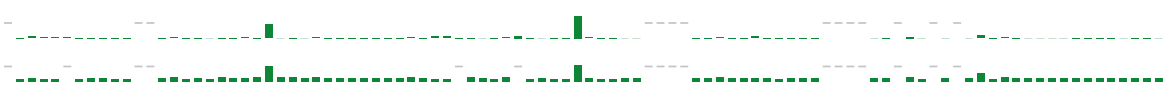

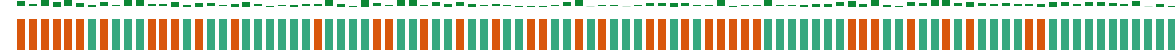
${ }_{0.9 \%}{ }^{*}|||||||||||||||||||||||||||||||||||||||||||||||||||||||||||||||||||||||||||||||||||||||||||||||||||||||||||||| \mid$

$\begin{array}{lllllllll}\mathrm{C}>\mathrm{A} & \mathrm{C}>\mathrm{G} & \mathrm{C}>\mathrm{T} & \mathrm{T}>\mathrm{A} & \| \mathrm{T}>\mathrm{C} & \| \mathrm{T}>\mathrm{G} & - \text { No data }\end{array}$

IYes - No Profiled for mutations $\quad$ Yes $\quad$ - No

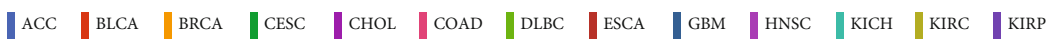

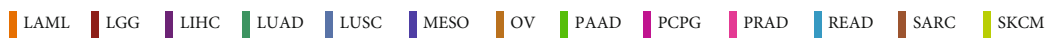

\begin{tabular}{l||l||l|l|l|l|l|l|} 
STAD & TGCT & THCA & THYM & UCEC & UCS & UVM
\end{tabular}

Yes - No MSIsensor score

No Yes MSI MANTIS score

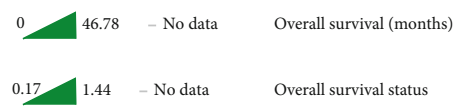

(b)

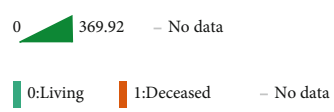

| 0:Living 1:Deceased - No data

Figure 4: Continued. 


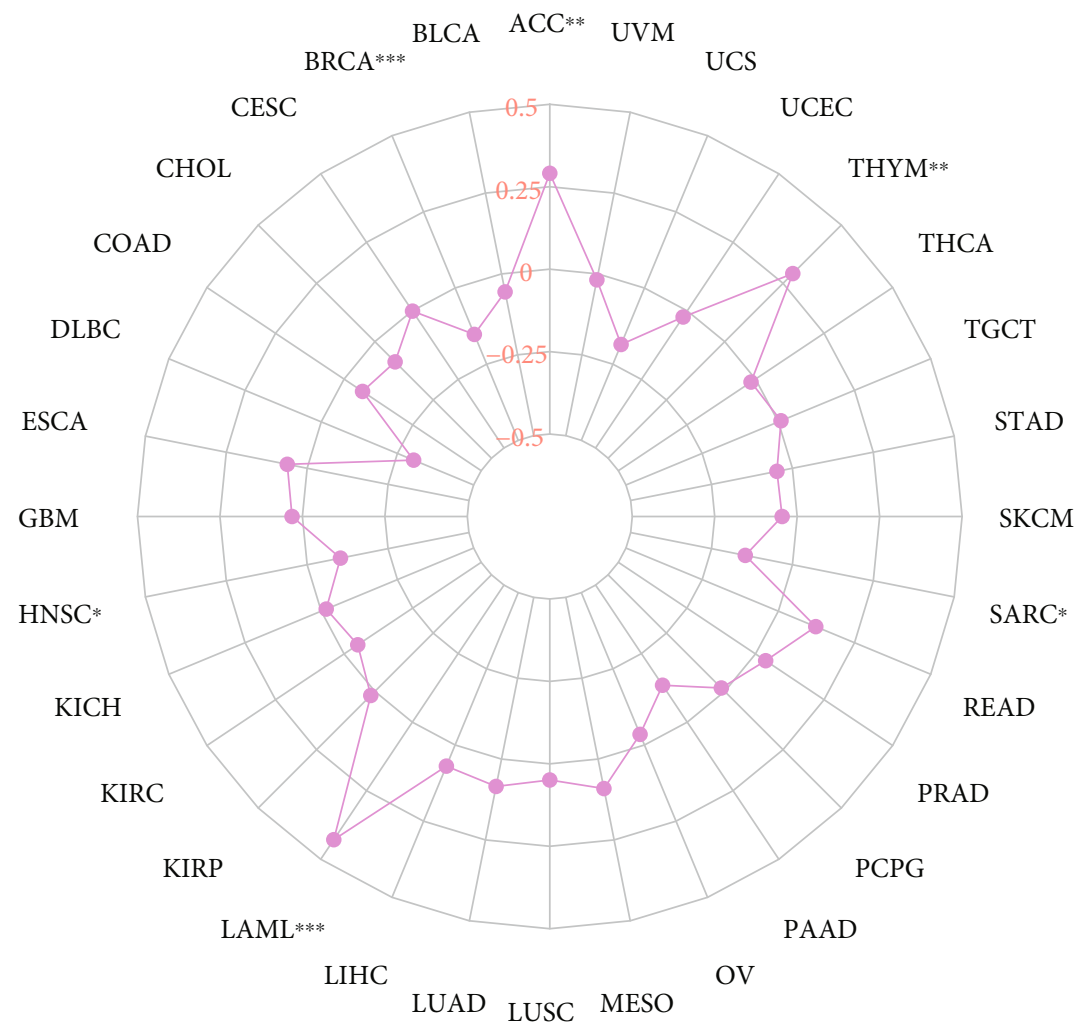

(c)

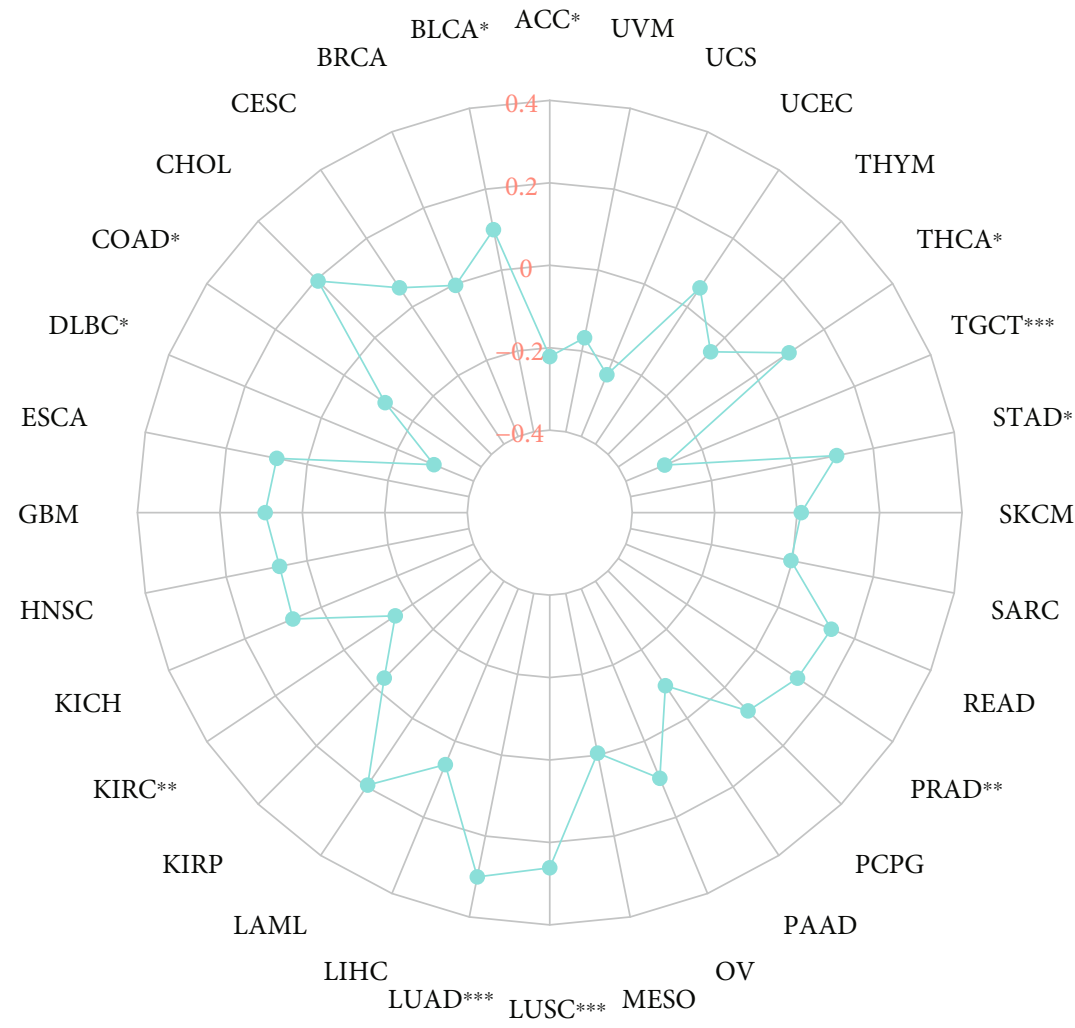

(d)

FIgURe 4: (a) Mutation characteristics of BACE1-AS in different carcinomas by using cBioPortal. (b) Detailed mutation information of BACE1-AS in mutated samples. (c) Correlation analysis of the expression level of TMB and BACE1-AS. (d) Correlation analysis of the expression level of MSI and BACE1-AS. ${ }^{*} P<0.05,{ }^{* *} P<0.01$, and ${ }^{* * *} P<0.001$. 
(Supplementary Figure 1a) indicated that highly expressed BACE1-AS correlated negatively with DFI in LIHC and HNSC, while the opposite results were found in PCPG and THCA. High BACE1-AS expression predicted poorer disease-specific survival (DSS) in KIRC and LIHC but longer DSS times in KICH, PAAD, and UVM (Supplementary Figure 1b). A univariate Cox regression analysis indicated that BACE1-AS is a protective factor in PAAD and $\mathrm{KICH}$ but is a risk factor in ACC, COAD, LIHC, and KIRC (Figures 2(c) and 2(d), Supplementary Figures $1 c$ and $1 d)$.

3.3. Clinical Characteristics of BACE1-AS in Pan-Cancer. The correlations between different clinical characteristics and BACE1-AS expression were further analyzed in 12 kinds of tumors with abnormal BACE1-AS expression. Considering that only one gender of PRAD, UCEC, and CESC was included in this study, no further analysis of the clinical characteristics of these three tumors was conducted. The results indicated that BACE1-AS expression levels in KIRP and LIHC (Figure 3(a)) were higher in patients younger than 65 years old $(P<0.05)$. BACE1-AS expression in male patients (Figure $3(\mathrm{~b})$ ) was higher than that in female patients only in KIRP $(P<0.05)$. In the other types of tumors, BACE1-AS expression was not significantly different between the genders. BACE1-AS expression levels were correlated with tumor stage in BRCA, KIRP, CHOL, COAD, and LIHC (Figure 3(c)). Generally, the higher the stage, the higher the expression level of BACE1-AS.

3.4. Mutation Analysis of BACE1-AS in Pan-Cancer. Figures 4(a) and 4(b) depict the BACE1-AS mutation results from TCGA cancer database following cBioPortal online analysis. The results revealed that the mutation forms of BACE1-AS in tumors were mainly amplification and deep deletion and that the mutation rate was highly focused in UVM and UCS. The correlation (Spearman) between BACE1-AS expression and tumor mutational burden (TMB) (Figure 4(c)) was significant in ACC, BRCA, LAML, SARC, HNSC, and THYM. Also, microsatellite instability (MSI) (Figure 4(d)) was significantly associated with BACE1-AS expression levels in BLCA, ACC, COAD, DLBC, KIRC, LUAD, LUSC, PRAD, STAD, TGCT, and THCA, all $P<0.05$. The correlation between BACE1-AS expression and tumor mutation load is shown in Table 2.

3.5. Correlation Analysis of BACE1-AS Expression and TME. We examined the correlation between BACE1-AS and TME (Figure 5) to evaluate the role of the BACE1-AS in tumor immunity. Accordingly, the tumors showing a significant correlation between BACE1-AS and immune scores in pan-cancers in TCGA database were BLCA, BRCA, CESC, COAD, GBM, KIRC, KIRP, LAML, LIHC, LUAD, LUSC, MESO, OV, PAAD, PCPG, PRAD, READ, SARC, STAD, TGCT, THCA, THYM, and UCEC (all $P<0.05$; Supplementary Figure 2). Generally, BACE1-AS expression levels were inversely correlated with immune scores, except for TGCT.
TABLE 2: Correlation between BACE1-AS expression and tumor mutation load.

\begin{tabular}{lcccc}
\hline Cancer type & \multicolumn{3}{c}{ TMB } & \multicolumn{2}{c}{ MSI } \\
\hline ACC & 0.29 & $<$ value & $R$ & $P$ value \\
BLCA & -0.06 & 0.26 & -0.22 & $<0.05^{* *}$ \\
BRCA & -0.15 & $<0.001^{* * *}$ & 0.10 & $<0.05^{*}$ \\
CESC & 0.00 & 0.99 & 0.06 & 0.92 \\
CHOL & -0.09 & 0.61 & 0.19 & 0.33 \\
COAD & -0.07 & 0.18 & -0.12 & $<0.25$ \\
DLBC & -0.30 & 0.07 & -0.30 & $<0.05^{*}$ \\
ESCA & 0.06 & 0.44 & 0.08 & 0.35 \\
GBM & 0.03 & 0.71 & 0.09 & 0.27 \\
HNSC & -0.10 & $<0.05^{*}$ & 0.07 & 0.13 \\
KICH & -0.02 & 0.90 & 0.07 & 0.56 \\
KIRC & -0.05 & 0.37 & -0.15 & $<0.01^{* *}$ \\
KIRP & 0.02 & 0.77 & -0.03 & 0.58 \\
LAML & 0.43 & $<0.001^{* * *}$ & 0.19 & 0.11 \\
LIHC & 0.07 & 0.19 & 0.06 & 0.24 \\
LUAD & 0.08 & 0.06 & 0.30 & $<0.001^{* * *}$ \\
LUSC & 0.05 & 0.28 & 0.26 & $<0.001^{* * *}$ \\
MESO & 0.09 & 0.42 & -0.01 & 0.96 \\
OV & -0.03 & 0.57 & 0.10 & 0.11 \\
PAAD & -0.13 & 0.10 & -0.10 & 0.21 \\
PCPG & -0.01 & 0.85 & 0.08 & 0.29 \\
PRAD & 0.04 & 0.42 & 0.12 & $<0.01^{* *}$ \\
READ & 0.12 & 0.16 & 0.14 & 0.09 \\
SARC & -0.15 & $<0.05^{*}$ & 0.00 & 0.95 \\
SKCM & -0.05 & 0.33 & 0.01 & 0.83 \\
STAD & -0.05 & 0.36 & 0.11 & $<0.05^{*}$ \\
TGCT & 0.01 & 0.92 & -0.30 & $<0.001^{* * *}$ \\
THCA & -0.02 & 0.72 & 0.10 & $<0.05^{*}$ \\
THYM & 0.29 & $<0.01^{* *}$ & -0.05 & 0.60 \\
UCEC & -0.02 & 0.61 & 0.06 & 0.20 \\
UVS & -0.19 & 0.17 & -0.24 & 0.08 \\
SHA & 0.87 & -0.17 & 0.14 \\
\hline & & & &
\end{tabular}

The correlations between BACE1-AS expression and stromal scores and estimate score are shown in Supplementary Figures 3 and 4 . The combined analysis of immune and stromal scores is denoted by the estimate scores. Both stromal and estimate scores were negatively correlated with BACE1-AS expression levels in tumors. The correlation between BACE1-AS and TME in GBM was the most significant, with BACE1-AS having the highest correlation coefficients with immune scores $(R=-0.53, P<0.05)$, stromal scores $(R=-0.51, P<0.05)$, and estimate scores $(R=-0.54$, $P<0.05)$.

3.6. Correlation Analysis of BACE1-AS with Immune Cell Infiltration in Pan-Cancer. Figure 6(a) indicates that the 


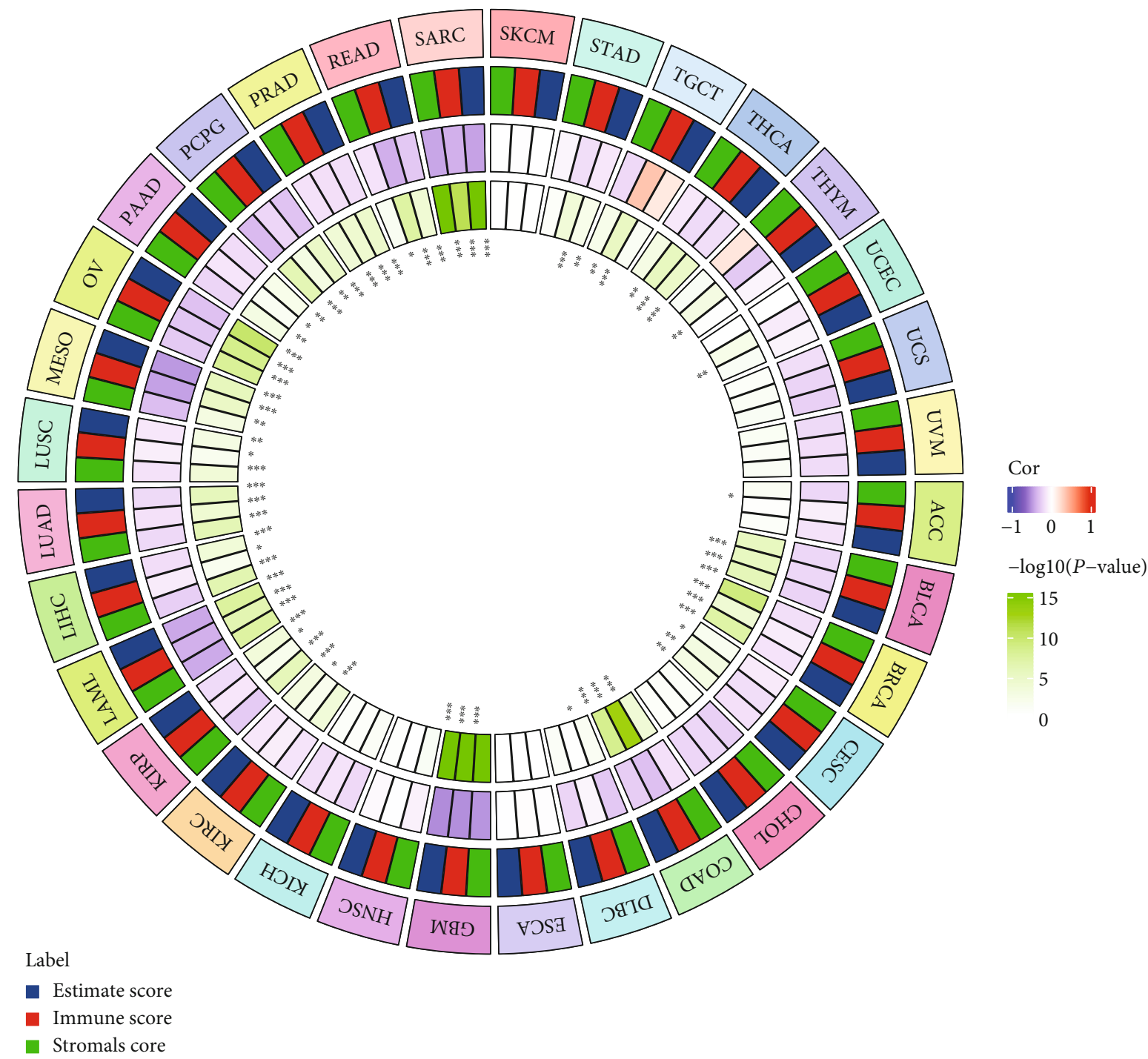

FIGURE 5: The correlation between the expression level of BACE1-AS and TME in pan-cancers. Association analysis of BACE1-AS with stromal score, immune score, and estimate score. ${ }^{*} P<0.05,{ }^{* *} P<0.01$, and ${ }^{* * *} P<0.001$.

strongest negative correlation is between T cells CD4 memory resting and $\mathrm{T}$ cells $\mathrm{CD} 8(R=-0.45, P<0.05)$, while monocytes and macrophages M2 have the most significant positive correlation $(R=0.39, P<0.05)$. The correlation analysis between 22 immune cell types and pan-cancer as based on the TIMER database is illustrated in Figures 6(b) and 7. Neutrophils, monocytes, regulatory $\mathrm{T}$ cells (Tregs), follicular $\mathrm{T}$ cells, activated memory $\mathrm{CD} 4 \mathrm{~T}$ cells, resting memory CD4 T cells, and naïve B cells were significantly associated with most tumors. Of all the infiltrating immune cells, neutrophils had the highest correlation in 10 tumor types including BLCA, COAD, HNSC, PAAD, SKCM, STAD, LUAD, OV, KIRC, and UCEC, indicating that the higher the BACE1-AS level, the lower the neutrophil content. Resting memory CD4 T cells were negatively correlated with ACC and KICH and positively correlated with THYM. Memory B cells were positively correlated with $\mathrm{CHOL}$ and negatively correlated with UCS. The highest correlations, both positive, with BRCA and LUSC were with follicular helper T cells. CESC and TGCT were most significantly negatively correlated with M2 macrophages, while monocytes were most significantly negatively correlated with LAML and PRAD. The strongest correlations with MESO, DLBC, THCA, READ, LIHC, KIRP, PCPG, SARC, GBM, and ESCA were with CD8 T cells, naïve B cells, activated dendritic cells, eosinophils, M0 macrophages, resting mast cells, activated natural killer (NK) cells, activated memory CD4 T cells, gamma delta $\mathrm{T}$ cells, and Tregs, respectively.

3.7. Correlation of BACE1-AS Expression with Immune Marker Sets. Immune checkpoints are one of the most promising targets for cancer therapy. They protect the immune system by preventing $\mathrm{T}$ cell overactivation from causing damage to the body. The upregulation of these immune marker sets can induce immune escape to inhibit the antitumor response of the immune system. Thus, we analyzed the relationship between 46 immune checkpoint genes and pan-cancer. 


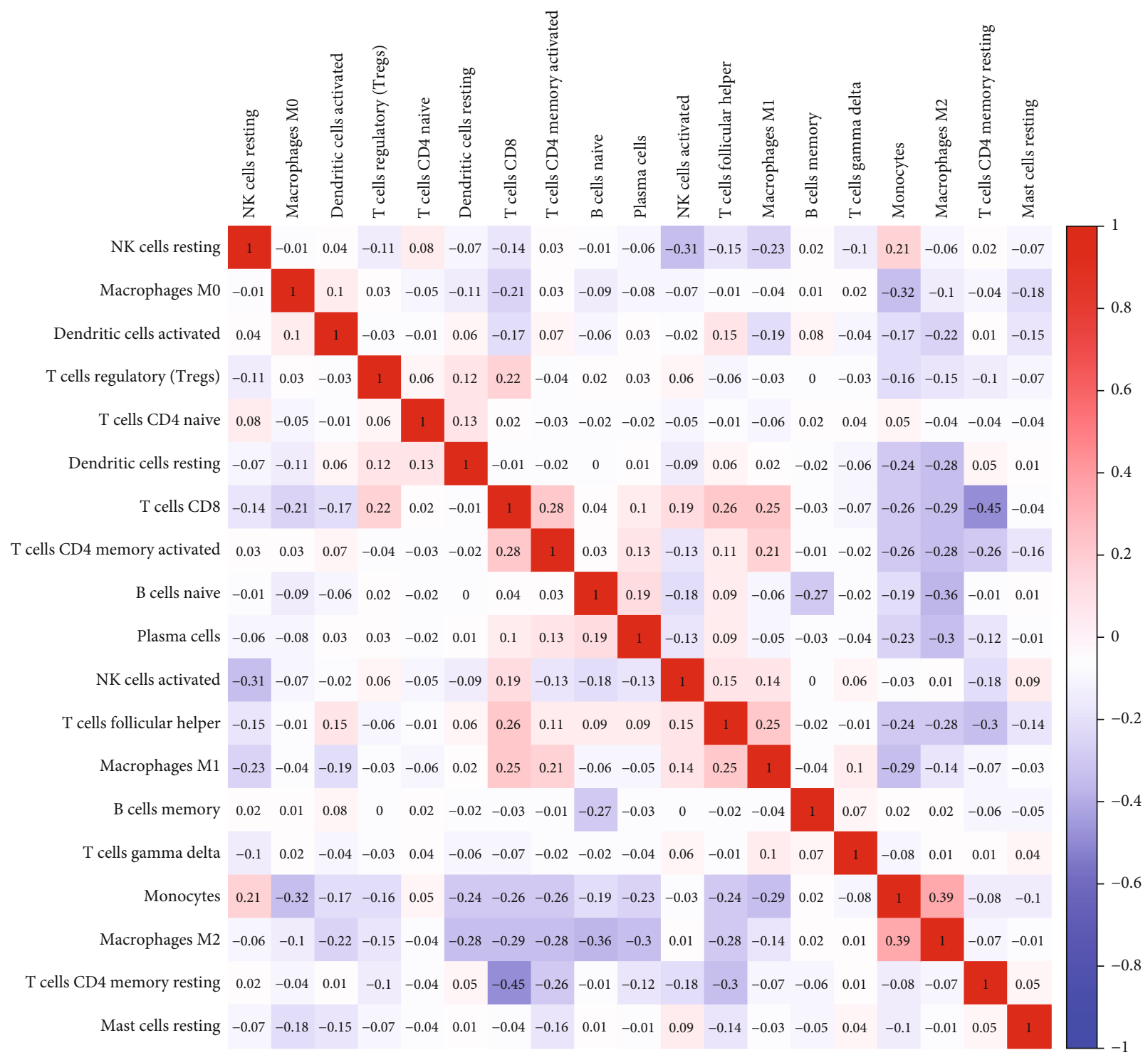

(a)

Figure 6: Continued. 


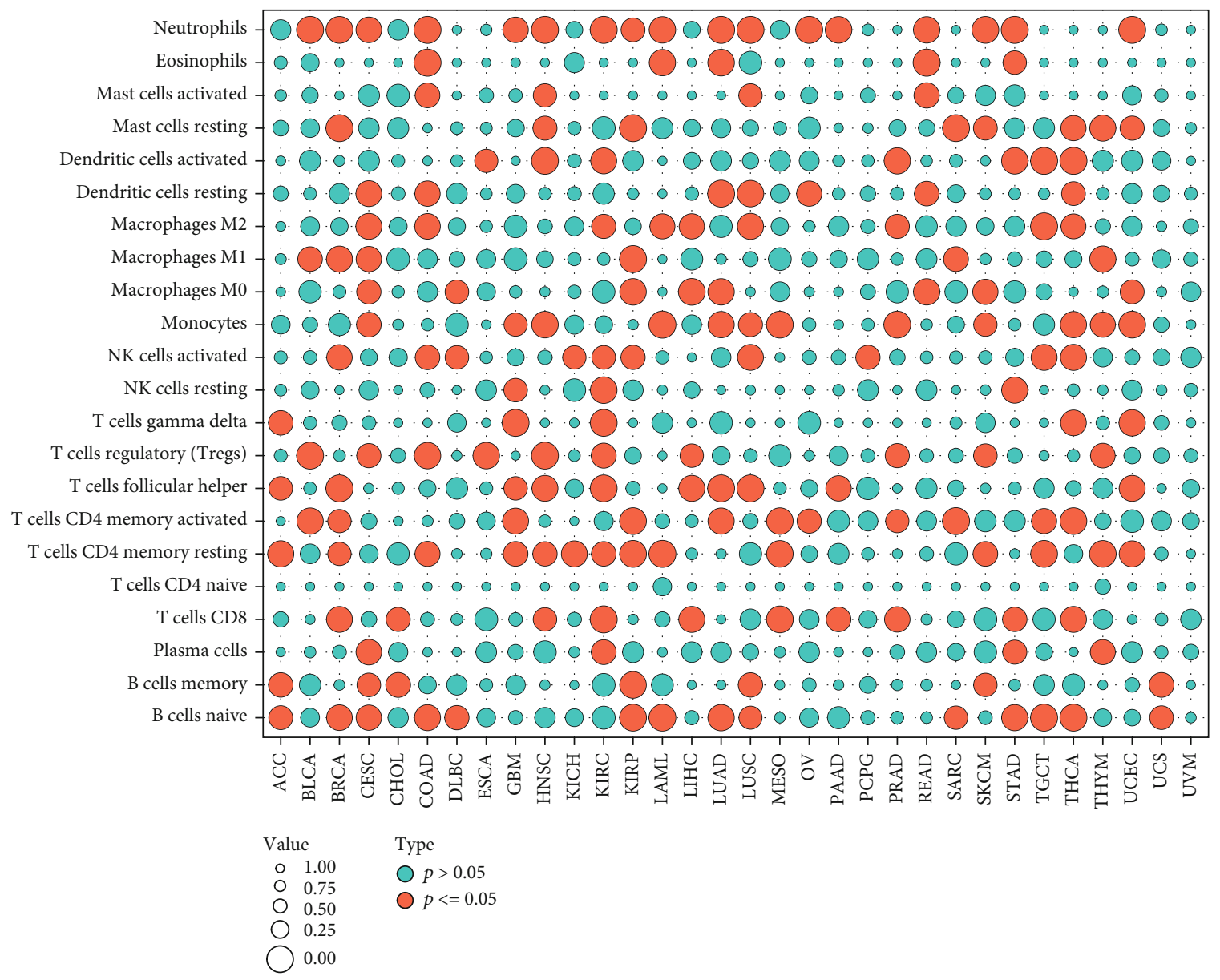

(b)

FIGURE 6: The correlation between the expression level of BACE1-AS and immune cell infiltration analysis in pan-cancers. (a) Correlation analysis between immune cells. The blue circle shows a negative correlation between the two genes, while the red circle shows a significant positive correlation. (b) Correlation of BACE1-AS expression with immune cells in pan-cancer. The red circles indicated BACE1-AS expression was significantly correlated with immune cells, while the blue circles indicated no correlation.

We found that THCA, TGCT, OV, GBM, and KIRC were the top five tumors significantly correlated with most immune marker genes (Figure 8). BACE1-AS expression had strong positive correlations with most of the immunomarker genes in TGCT, CHOL, HNSC, KIRC, and LIHC. Further, TNFRSF25, PDCD1LG2, CD276, CD80, HAVCR2, LAIR1, CD160, TNFRSF14, ADORA2A, and CD48 were significantly associated with BACE1-AS expression in more than half of the tumor types. Notably, KIR3DL1 was the gene negatively correlated with BACE1-AS expression only in PRAD $(R=-0.10, P<0.05)$. ACC was significantly correlated with TNFRSF25 $(R=0.34, P<0.05)$ and TNFRSF14 $(R=0.22, P<0.05)$ only. Finally, DLBC was significantly correlated with CD276 $(R=-0.34, P<0.05)$ and BTNL2 $(R=0.33, P<0.05)$.

3.8. Gene Set Enrichment Analysis in the Low- and HighExpression BACE1-AS Groups. We applied Gene Ontology (GO) and Kyoto Encyclopedia of Genes and Genomes (KEGG) enrichment analysis on different BACE1-AS expression levels in pan-cancer. We found that KEGG func- tion between the high- and low-expression BACE1-AS groups was significantly different in 15 types of tumors and was significantly different in GO enrichment across all tumor types.

BACE1-AS expression mainly affected pathways including complement and coagulation cascades, RRAR signaling pathway, intestinal immune network for immunoglobulin A (IgA) production, cancer pathways, autophagy regulation, cytokine-cytokine receptor interaction, and antigen processing and presentation, which are mainly related to tumorigenesis and immune activity (Figure 9). The result of the GO analysis is shown in Supplementary Figure 5.

3.9. Expression Characteristics and Role of BACE1-AS in Hepatocellular Carcinoma (HCC). The role of BACE1-AS in tumorigenesis and its relationship with immunity in pan-cancer cannot be ignored by the above analysis. However, experimental evidence is lacking. We further validated the role of BACE1-AS in HCC at cellular levels using quantitative real-time polymerase chain reaction (qRT-PCR). Figure 10(f) shows that BACE1-AS expression levels were 


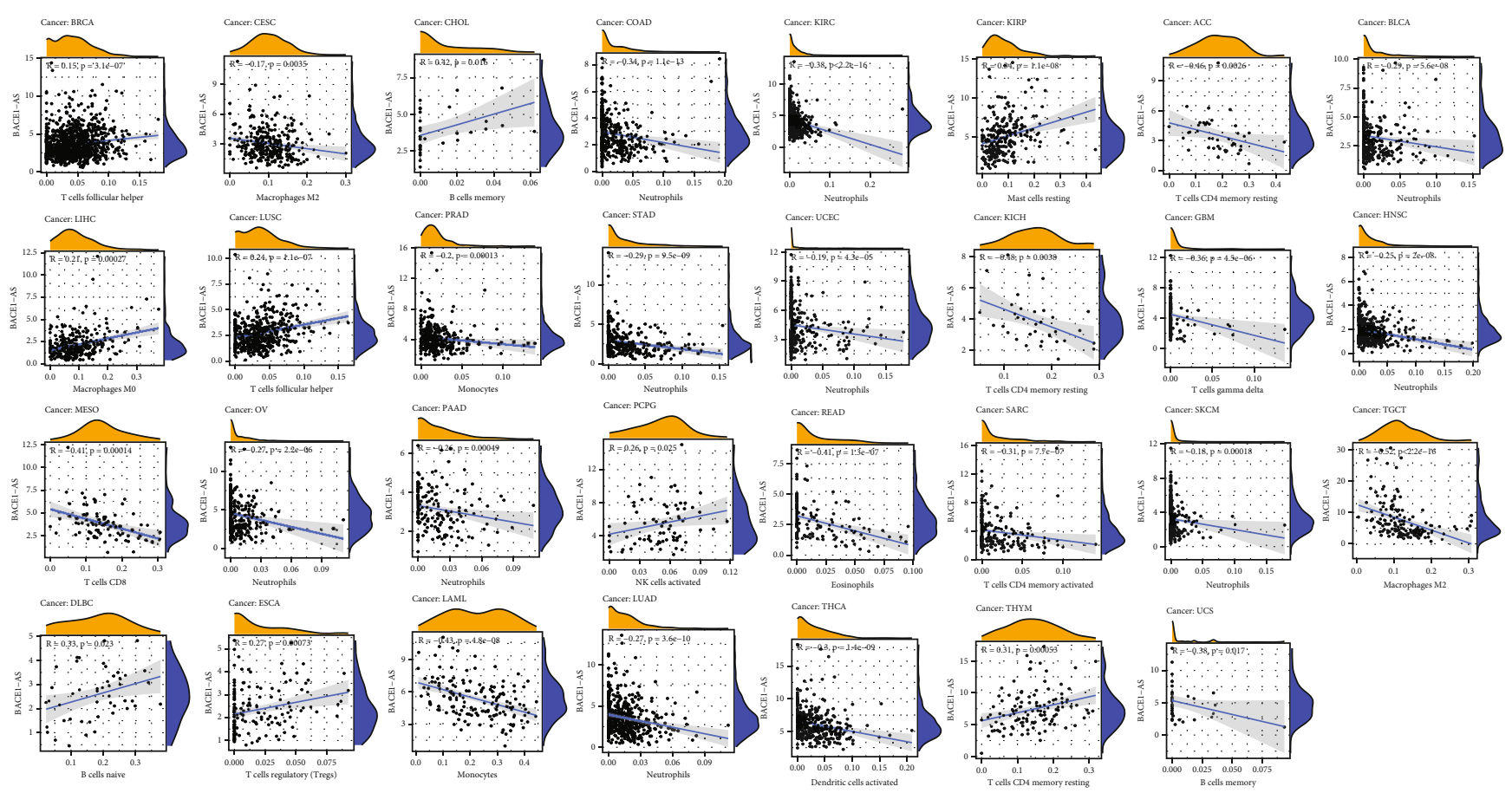

FIGURE 7: Correlation of BACE1-AS expression with immune cells in pan-cancer. Only the most closely related tumor infiltrating cells were exported in the figure.

significantly increased in the Hep3B, HLE, and HLF cell lines compared to the normal liver cell line LO2 $(P<0.05)$.

19 immune checkpoint genes (CD276, TNFRSF14, ADORA2A, TNFSF15, BTNL2, TNFRSF4, PDCD1, TNFSF4, NRP1, TNFRSF8, TNFRSF25, LGALS9, HHLA2, TNFSF14, TNFSF9, TNFRSF18, VTCN1, CD70, and CTLA4) were identified significantly associated with BACE1-AS expression (Figure 8). To further verify the interaction between BACE1-AS and immune checkpoint genes in tumors, a prognostic model using Cox regression analysis was established using BACE1-AS and NRP1, an immune checkpoint gene coexpressed with BACE1-AS. Figure 10(c) indicates that the model can predict the prognosis of HCC patients independently of other clinical factors. The area under the time-dependent curve (AUC) of 1-, 2-, and 3-year survival in the risk model was 0.684, 0.620, and 0.607 (Figure 10(e)). The patients were divided into risk groups according to their median risk score. In the low-risk group, more patients survived with higher survival rates, and BACE1-AS and NRP1 were more highly expressed (Figures 10(b) and 10(d)). By analyzing the effect of NRP1 and BACE1-AS expression levels on patient survival, it was found that patients with high expression levels of both NRP1 and BACE1-AS had the lowest survival rate. In contrast, survival rate was the highest when both NRP1B and BACE1-As expression levels were low (Figure 10(a)). Moreover, this two-gene signature model can significantly predict the sensitivity of common chemotherapeutic drugs (Figure 10(g)); for example, higher IC50s and lower sensitivities were predicted in axitinib, docetaxel, erlotinib, methotrexate, sorafenib, and sunitinib in high-risk patients, while a lower IC50 and higher sensitivity of doxorubicin were predicted in high-risk patients (all $P<0.05)$.

\section{Discussion}

BACE1-AS, a conserved noncoding antisense transcript for BACE1 that can bind to BACE1 mRNA to improve its stability, was reported to be upregulated and a potential biomarker for Alzheimer's disease [12, 13]. Notably, the expression level of BACE1-AS has been reported to play an important role in the progression of gastric cancer [14]. Moreover, anisomycin can inhibit the proliferation and invasion of ovarian cancer stem cells by increasing BACE1-AS levels [21]. Using bioinformatics, Nie et al. identified BACE1-AS as a poor prognostic factor for HCC [22]. Here, we found that BACE1-AS was highly expressed in most tumor types, and its abnormal expression affected the survival times of some tumors. Moreover, from our qRTPCR experiment, the expression levels of BACE1-AS in three HCC cell lines were higher than in LO2. Our results are highly consistent with previous studies. Altogether, the aforementioned direct or indirect data suggest that the role of BACE1-AS in tumors cannot be ignored.

With the development of molecular biology, great progress has been made in the study of tumor etiology and pathogenesis. However, the occurrence and development of tumors are extremely complex with multiple factors and multiple steps. More specifically, the occurrence of tumors is not simply the result of a single gene mutation; it is a long-term, phasic, accumulative process involving multiple 


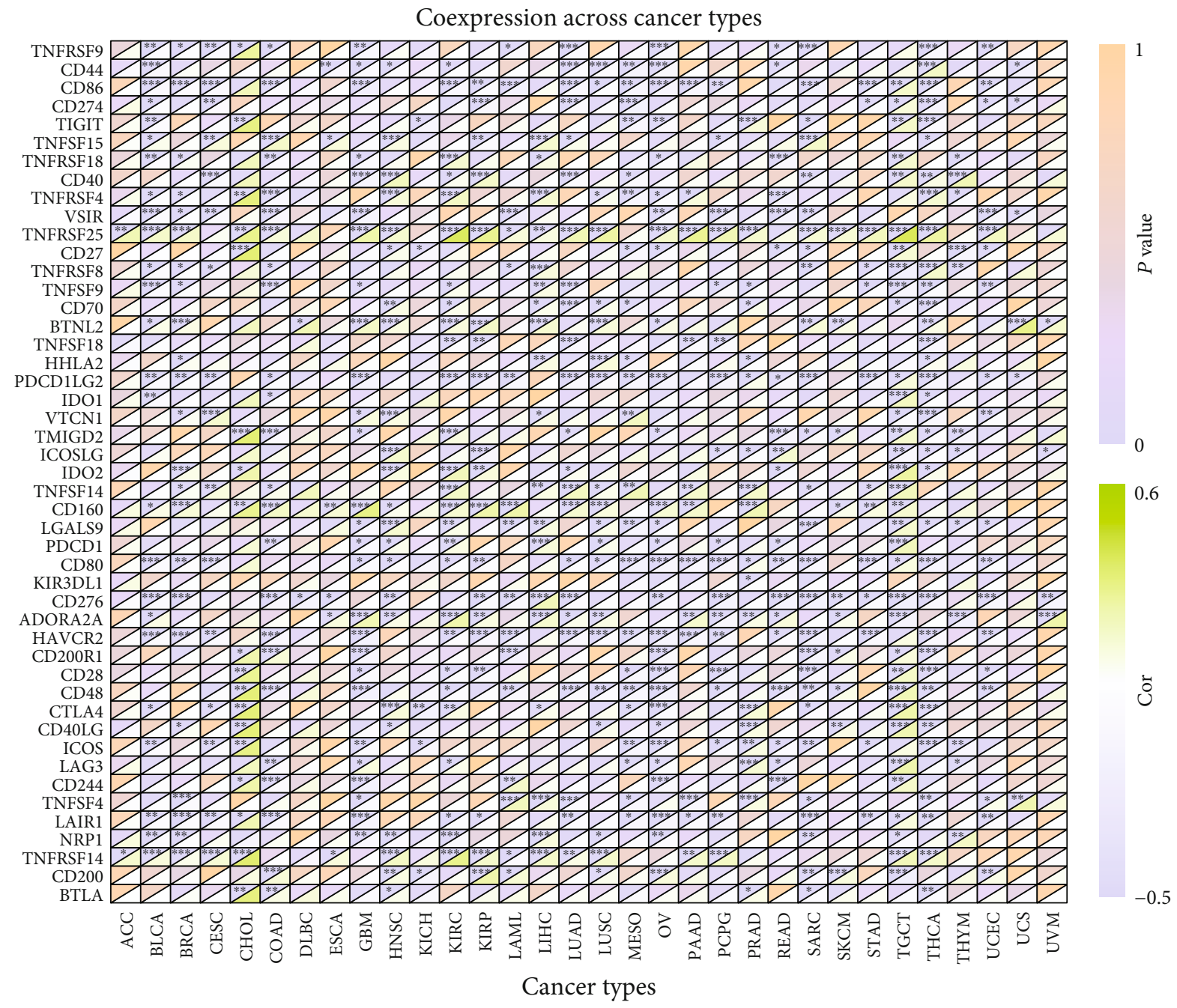

Figure 8: Heat map of correlation analysis between BACE1-AS expression and immune marker sets. ${ }^{*} P<0.05,{ }^{* *} P<0.01$, and ${ }^{* * *} P<0.001$.

gene mutations $[1,4]$. We reported that TMB was significant in ACC, BRCA, LAML, SARC, HNSC, and THYM and that MSI was significantly associated with BACE1-AS expression levels in 11 types of tumors. These findings indicate the potential mechanisms of BACE1-AS in mediating tumorigenesis from the perspective of tumor mutation.

TME, a fundamental direction in tumor research, plays an important role in the diagnosis, prevention, and prognosis of tumors; it is of great significance in understanding tumor occurrence, development, and metastasis [23]. Presently, it is believed that the body's immune surveillance system plays an important role in preventing the occurrence of tumors; thus, the occurrence of tumors may result from the loss of immune surveillance $[24,25]$. Our study is the first to analyze the relationship between BACE1-AS expression and tumor immunity of pan-cancer, including immune microenvironment, infiltrating immune cells, and immune checkpoint targets. BACE1-AS expression was correlated with immune scores in 23 types of tumors: it was negatively correlated with 22 types and positively correlated with TCGT. The higher the expression of BACE1-AS, the smaller the proportions of stromal and estimate scores in pan-cancer.
We know that the higher the three immune scores, the higher the content of immune cells in the tumor sample and the lower the purity of the tumor cells. In other words, tumor cells are fewer when BACE1-AS expression is low. This finding highly corresponds with the higher expression of BACE1-AS in LIHC, KIRP, KIRC, CHOL, STAD, KICH, COAD, and PRAD compared to that in normal tissues. The analysis of immune components in the TME can help increase the understanding of how immune composition and immune status can affect cancer cells and cancer treatment. To some extent, the occurrence of tumors is an abnormal inflammatory response. Tumor cells recruit inflammatory cells to reach tumor tissues by producing various inflammatory factors, such as growth factors, cytokines, and chemokines $[26,27]$.

Immune infiltrating cells in tumor tissues can change the metabolism and function of tumor cells and promote tumor immunosuppression and immune escape [28]. We analyzed 22 types of effector cells involved in the tumor immune response in pan-cancer. We found that nine immuneassociated cells were significantly associated with BACE1AS expression levels in more than 10 types of tumors 

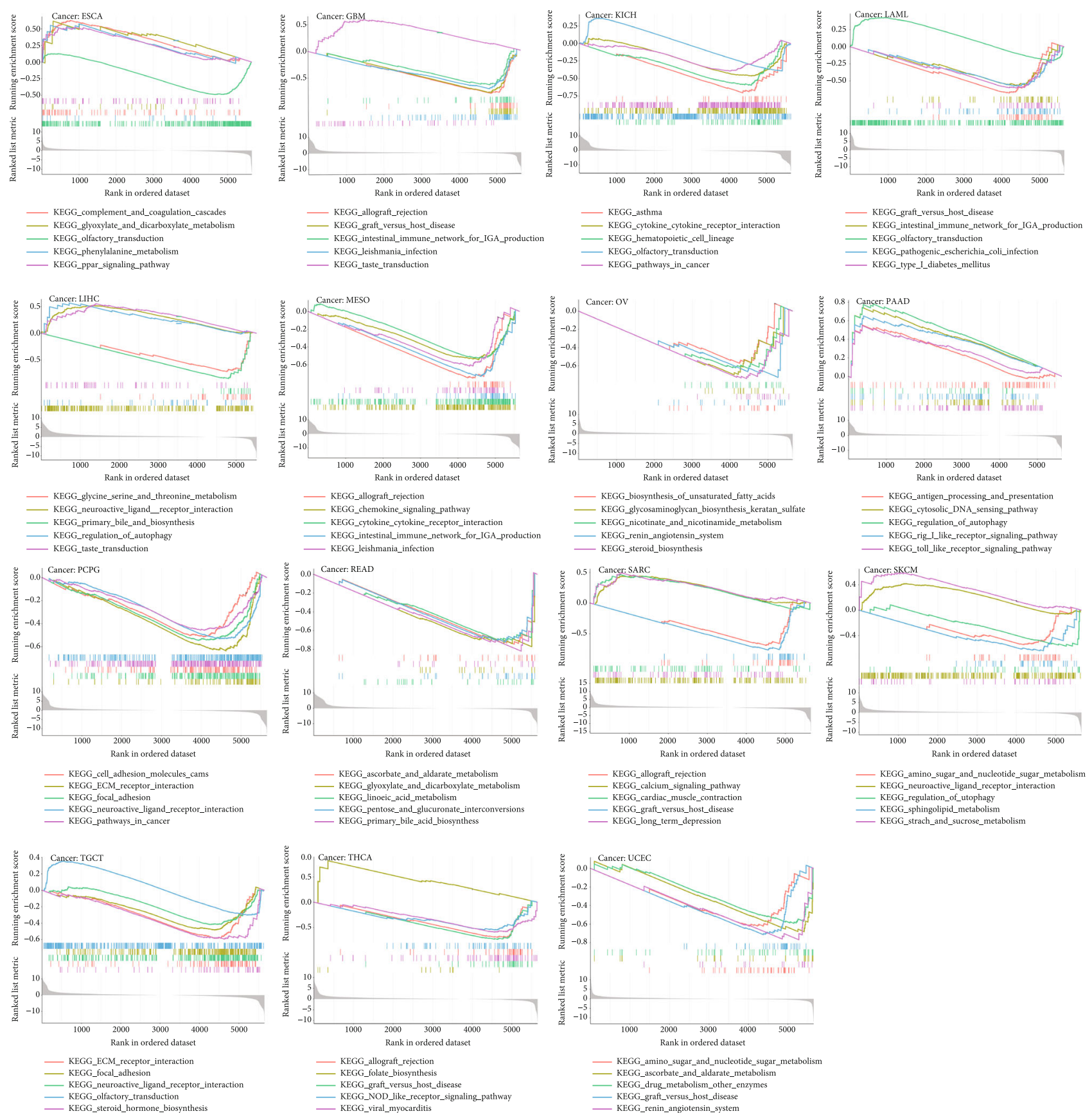

FIGURE 9: KEGG analysis of high and low expression of BACE1-AS in pan-cancer. Each of the different colored lines represents a specific gene set. The upregulated genes were showed at the left side near the origin, while the downregulated gene set was at the right side. Only $P$ value $<0.05$ was considered significant.

$(P<0.05)$. However, the expression levels of BACE1-AS in UVM were not significantly correlated with these immune cells. Neutrophils were significantly negatively correlated with BACE1-AS expression in the most common tumor types. Notably, infiltrating neutrophils may promote tumor-associated inflammation or, through the expression of antitumor and cytotoxic mediators, inhibit tumor growth [29]. This characteristic of neutrophils may explain why most tumors are more aggressive and have poorer prognosis when BACE1-AS expression is upregulated. NK cells and
BACE1-AS expression levels were negatively correlated in four tumor types and positively correlated in six. NK cells play a vital role in antitumor immunity by directly recognizing and killing tumor cells. Even tumor cells that develop strategies to evade recognition by CD8+ T cells are attacked by NK cells [30]. We also found that BACE1-AS expression levels were negatively correlated with Tregs in two types of tumors and positively correlated in eight types, indicating that BACE1-AS may be involved in the regulation of tumor immunity. Macrophages can play an immunosuppressive 


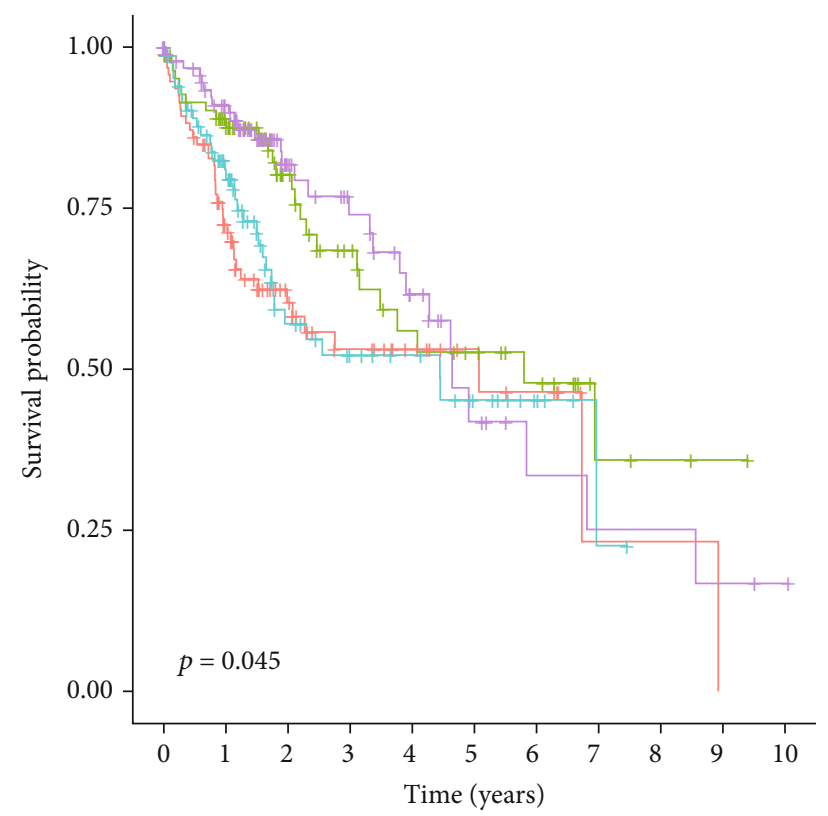

Type

- NRP1 high + BACE1-AS high

+ NRP1 high + BACE1-AS low

+ NRP1 low + BACE1-AS high

+ NRP1 low + BACE1-AS low

(a)
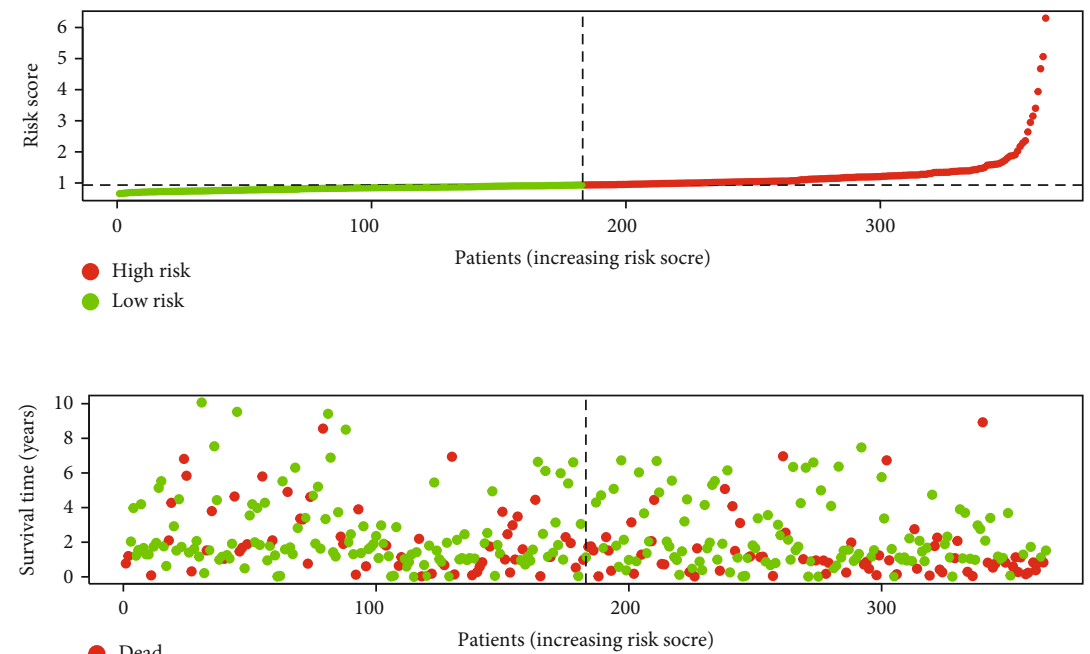

Dead

Patients (increasing risk socre)

Alive

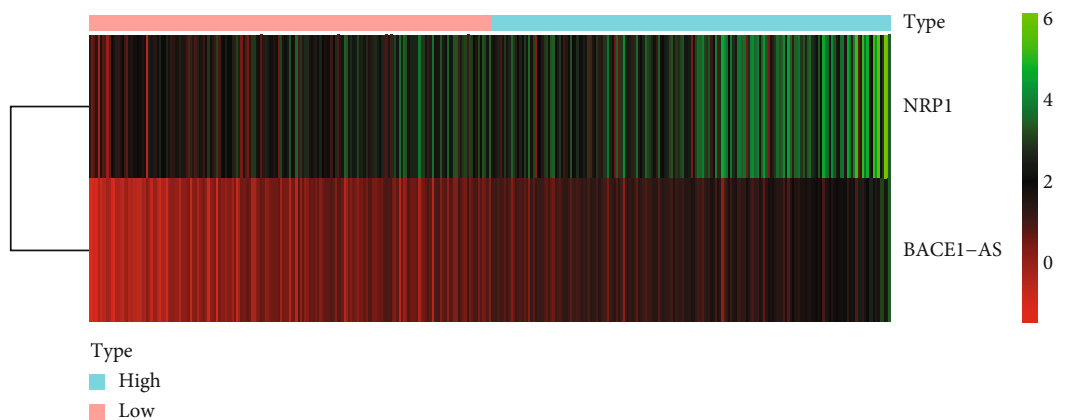

(b)

Figure 10: Continued. 


$\begin{array}{lrr} & p \text { value } & \text { Hazard ratio } \\ \text { Age } & 0.591 & 1.005(0.987-1.023) \\ \text { Gender } & 0.301 & 0.780(0.487-1.249) \\ \text { Grade } & 0.914 & 1.017(0.746-1.387) \\ \text { Stage } & <0.001 & 1.865(1.456-2.388) \\ \text { T } & <0.001 & 1.804(1.434-2.270) \\ \mathrm{M} & 0.023 & 3.850(1.207-12.281) \\ \mathrm{N} & 0.328 & 2.022(0.494-8.276) \\ \text { risk } & 0.002 & 1.430(1.144-1.787)\end{array}$

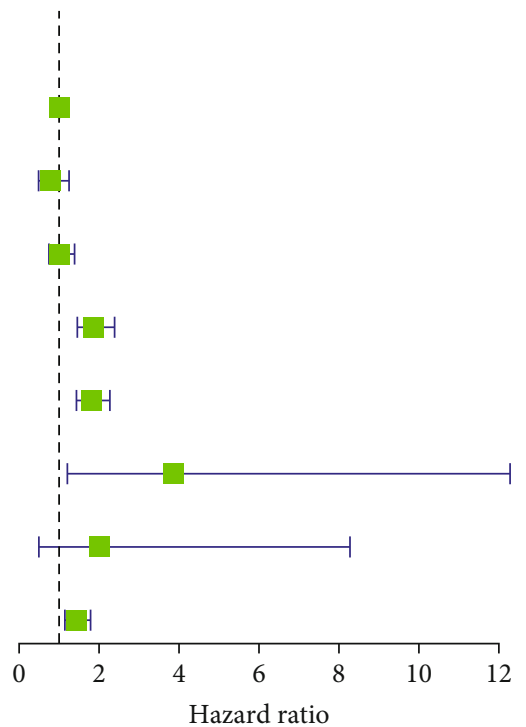

(c)
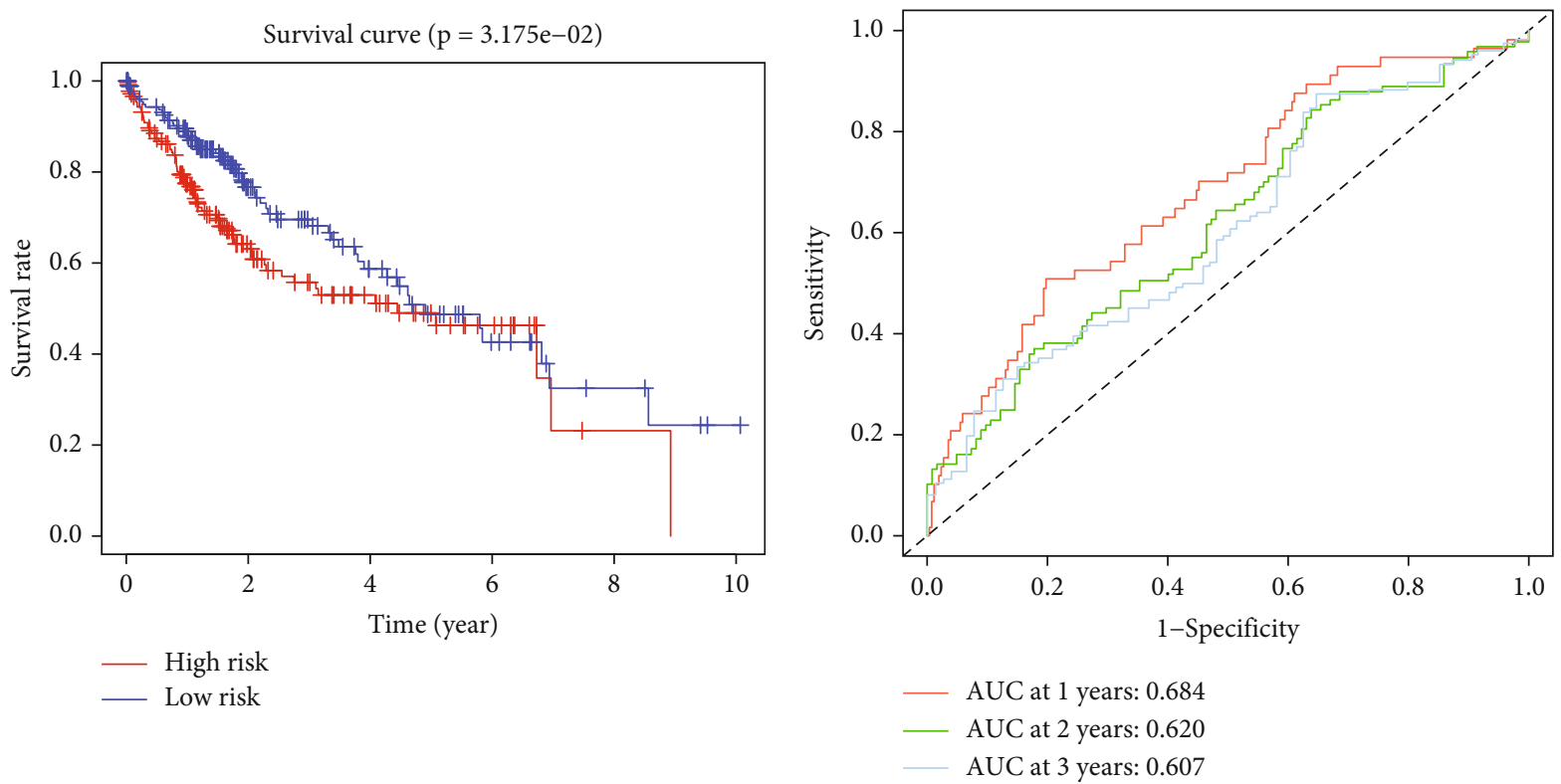

(d)

(e)

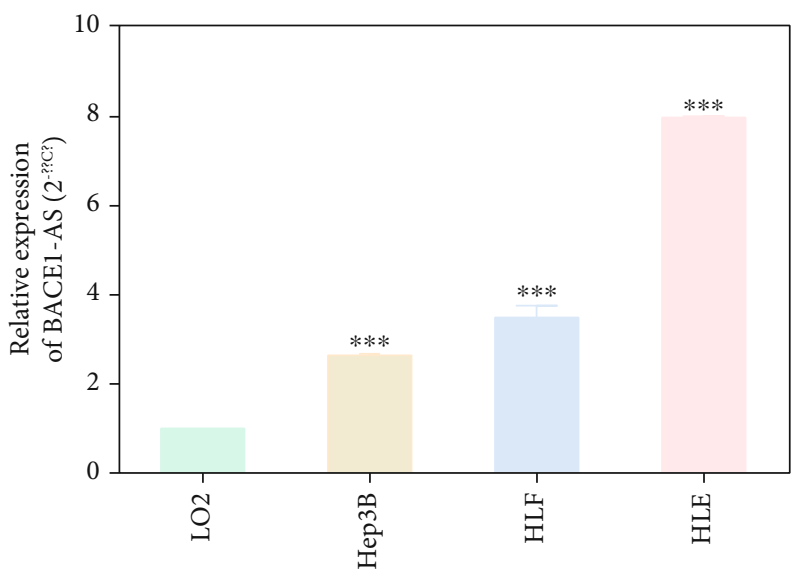

(f)

Figure 10: Continued. 

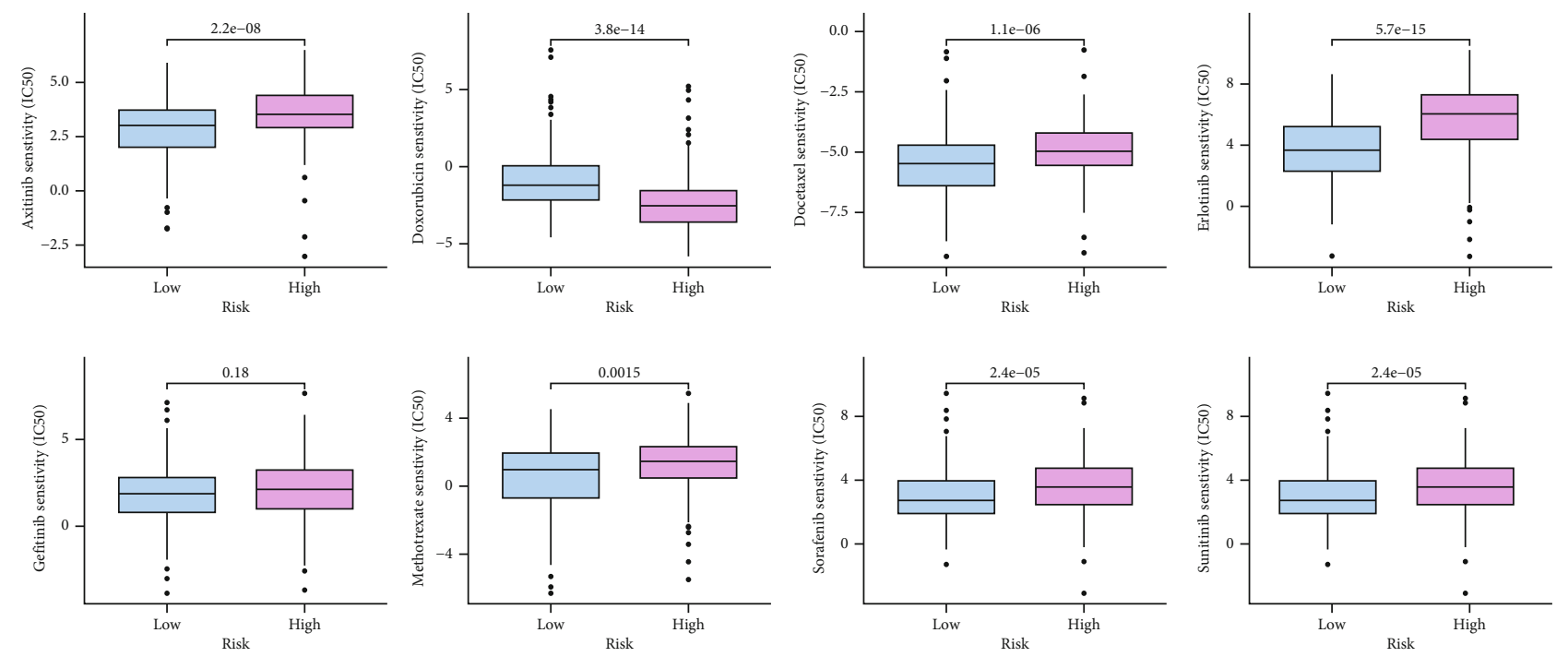

Risk
白 Low
白 High

(g)

FIGURE 10: Validation of the role of BACE1-AS in hepatocellular carcinoma. (a) The combined effect of BACE1-AS and NRP1 on the prognosis of hepatocellular carcinoma. (b) Analysis of survival status and gene expression in high- and low-risk patients. (c) Independent prognostic analysis of the 2-gene signature model risk score. (d) Prognosis difference between high- and low-risk groups of HCC patients. (e) Area under the curve of the risk model at 1, 2, and 3 years. (f) Expression level of BACE1-AS in normal liver cells and liver cancer cells. (g) Evaluation of the effect of risk model on drug sensitivity.

role via the PI3K $\gamma$ signaling pathway. Based on their phenotypes and functions, macrophages can be divided into classically activated macrophages (M1 type) and alternatively activated macrophages (M2 type), also known as tumorassociated macrophages (TAMS). TAMs can prevent T cells from attacking tumor cells, secrete growth factors to nourish tumor cells, promote the formation of blood vessels in tumor tissues, and further promote the metastasis and proliferation of tumor cells [31, 32]. We found that M2 macrophages were negatively correlated with BACE1-AS in TGCT, LIHC, LUCC, COAD, PRAD, and KIRC and positively correlated with BACE1-AS in THCA, CESC, and LAML. These results suggest that BACE1-AS may play an immunosuppressive role in cancer by promoting the presentation of tumor antigens.

Immune checkpoint molecules, such as CTLA4, NRP1, TNFSF15, CD44, and BTLA, are present in the TME or on the surface of tumor cells, and they negatively regulate $\mathrm{T}$ cell activation. Under normal conditions, these molecules mainly maintain $\mathrm{T}$ cell homeostasis, but during tumor evolution, these molecules can help tumor cells escape the immune response $[33,34]$. CTLA4 and PD-L1 (CD274) are two relatively mature immune checkpoint molecules. CTLA4 and BACE1-AS expression was significantly positively correlated in seven types of tumors and negatively correlated in six, while CD274 was significantly correlated with BACE1-AS expression in 10 types of tumors. This data not only further reveal the potential association between BACE1-AS and tumor immunity but also suggest that we can further experimentally verify the effectiveness of immu- nosuppressive therapies in relevant cancer types. TNFRSF25, a $T$ cell costimulatory molecule, which we found to be significantly associated with BACE1-AS expression among 23 types of tumors, is correlated with the pathogenesis of liver cancer, pancreatic cancer, breast cancer, colon cancer, and leukemia and activates the NF- $\kappa \mathrm{B}$ and MARK signaling pathways [35-39]. Thus, TNFRSF25 may be a key immune checkpoint molecule mediating the effect of BACE1-AS on tumor progression, and further study on the association between the two may help to develop new therapeutic targets. The results of the prognostic model on HCC prognosis and chemosensitivity as constructed using BACE1-AS and NRP1 further confirm the close relationship between BACE1-AS and tumor immunity in tumor progression.

We found that BACE1-AS expression affects pathways related to tumorigenesis and immune activity. These pathways include the complement and coagulation cascades, RRAR signaling pathway, intestinal immune network for IgA production, cancer pathways, autophagy regulation, cytokine-cytokine receptor interaction, and antigen processing and presentation. These results account for the association between BACE1-AS expression and tumor immunity from the perspective of internal mechanisms, but further experimental investigation is needed for verification.

Overall, our results suggest that BACE1-AS is abnormally expressed in most tumor types, and its expression level is correlated with clinical features, prognosis, TMB, and MSI. Moreover, BACE1-AS expression may affect the TME, the content of infiltrating immune cells, the immune molecular checkpoints, and the immune activity-related 
pathways. Altogether, our data indicate that BACE1-AS can be a potential immunotherapy target in improving cancer patient outcomes.

\section{Data Availability}

The datasets presented in this study can be found in online repositories. The names of the repository/repositories and accession number(s) can be found in the article.

\section{Conflicts of Interest}

The authors declare that the research was conducted in the absence of any commercial or financial relationships that could be construed as a potential conflict of interest.

\section{Acknowledgments}

The work was supported by the diagnostic criteria and comprehensive intervention study on the elderly frailty in China, the National Key Research and Development Program of China (2018YFC2002000).

\section{Supplementary Materials}

Supplementary 1. Supplementary Figure 1: prognostic value of BACE1-AS in different tumors. (a, b) Kaplan-Meier curves estimate the DFI and DSS differences in pan-cancer. (c, d) DFI and DSS differences among pan-cancer analyzed by univariate regression analysis. Only survival curves with significant differences $(P<0.05)$ were shown.

Supplementary 2. Supplementary Figure 2: correlation difference of BACE1-AS expression and immune score in tumors with $P<0.05$ was shown. ${ }^{*} P<0.05,{ }^{* *} P<0.01$, and ${ }^{* * *} P<0.001$.

Supplementary 3. Supplementary Figure 3: the correlation between BACE1-AS expression and stromal score. The stromal scores were negatively correlated with BACE1-AS expression levels in tumors with $P$ value $<0.05$ shown.

Supplementary 4. Supplementary Figure 4: relationship between expression level of BACE1-AS and tumor estimate score. The figure shows a significant correlation between the types of tumors. Tumor types with a significant negative correlation with BACE1-AS expression were shown in the figure.

Supplementary 5. Supplementary Figure 5: GO analysis reveals the molecular functions related to the expression level of BACE1-AS. Different colored curves represent different functions. The peak value of the curve at the top and in the part of positive gene correlation indicates that molecular functions here were positively correlated with BACE1-AS expression.

\section{References}

[1] P. Vineis and C. P. Wild, "Global cancer patterns: causes and prevention,” Lancet, vol. 383, no. 9916, pp. 549-557, 2014.
[2] J. Ferlay, M. Colombet, I. Soerjomataram et al., "Cancer statistics for the year 2020: an overview," International Journal of Cancer, vol. 149, no. 4, pp. 778-789, 2021.

[3] H. Sung, J. Ferlay, R. L. Siegel et al., "Global cancer statistics 2020: GLOBOCAN estimates of incidence and mortality worldwide for 36 cancers in 185 countries," Ca-a Cancer Journal for Clinicians, vol. 71, no. 3, pp. 209-249, 2021.

[4] B. Vogelstein and K. W. Kinzler, "The multistep nature of cancer," Trends in Genetics, vol. 9, no. 4, pp. 138-141, 1993.

[5] D. C. Hinshaw and L. A. Shevde, "The tumor microenvironment innately modulates cancer progression," Cancer Research, vol. 79, no. 18, pp. 4557-4566, 2019.

[6] L. Incorvaia, D. Fanale, G. Badalamenti et al., "Programmed death ligand 1 (PD-L1) as a predictive biomarker for pembrolizumab therapy in patients with advanced non-small-cell lung cancer (NSCLC)," Advances in Therapy, vol. 36, no. 10, pp. 2600-2617, 2019.

[7] R. J. Kishton, M. Sukumar, and N. P. Restifo, "Metabolic regulation of $\mathrm{T}$ cell longevity and function in tumor immunotherapy," Cell Metabolism, vol. 26, no. 1, pp. 94-109, 2017.

[8] W. X. Peng, P. Koirala, and Y. Y. Mo, "LncRNA-mediated regulation of cell signaling in cancer," Oncogene, vol. 36, no. 41, pp. 5661-5667, 2017.

[9] J. L. C. Richard and P. J. A. Eichhorn, "Platforms for investigating LncRNA functions," Slas Technology, vol. 23, no. 6, pp. 493-506, 2018.

[10] J. K. Huang, L. Ma, W. H. Song et al., "LncRNA-MALAT1 promotes angiogenesis of thyroid cancer by modulating tumor-associated macrophage FGF2 protein secretion," Journal of Cellular Biochemistry, vol. 118, no. 12, pp. 4821-4830, 2017.

[11] J. Cao, R. Dong, L. Jiang et al., "LncRNA-MM2P identified as a modulator of macrophage M2 polarization," Cancer Immunology Research, vol. 7, no. 2, pp. 292-305, 2019.

[12] M. A. Faghihi, F. Modarresi, A. M. Khalil et al., "Expression of a noncoding RNA is elevated in Alzheimer's disease and drives rapid feed-forward regulation of $\beta$-secretase," Nature Medicine, vol. 14, no. 7, pp. 723-730, 2008.

[13] Y. Zhou, Y. Ge, Q. Liu et al., "LncRNA BACE1-AS promotes autophagy-mediated neuronal damage through the miR-2143p/ATG5 signalling axis in Alzheimer's disease," Neuroscience, vol. 455, pp. 52-64, 2021.

[14] F. Esfandi, S. Ghafouri-Fard, V. K. Oskooei, and M. Taheri, “ $\beta$ Secretase 1 and its naturally occurring anti-sense RNA are down-regulated in gastric cancer," Pathology \& Oncology Research, vol. 25, no. 4, pp. 1627-1633, 2019.

[15] F. Farris, V. Matafora, and A. Bachi, "The emerging role of $\beta$ secretases in cancer," Journal of Experimental \& Clinical Cancer Research, vol. 40, no. 1, 2021.

[16] H. Yaghoobi, H. Azizi, M. Banitalebi-Dehkordi et al., "Betasecretase $1(B A C E)$ is down-regulated in invasive ductal carcinoma of breast," Reports of Biochemistry \& Molecular Biology, vol. 8, no. 2, pp. 200-207, 2019.

[17] C. Yuan, Y. Wei, L. Hu, Y. Tian, and Y. Liu, "MiR-574-3p accelerates cell migration and invasion through regulating BACE1 in non-small cell lung cancer," Panminerva Medica, 2019.

[18] K. Zhai, Z. Huang, W. Tao, X. Fang, and S. Bao, Inhibition of BACE1 Facilitates Macrophage-Based Immunotherapy to Suppress Malignant Growth of Glioblastoma, Research Square, 2020. 
[19] L. Wei, Z. Jin, S. Yang, Y. Xu, Y. Zhu, and Y. Ji, “TCGAAssembler 2: software pipeline for retrieval and processing of TCGA/CPTAC data," Bioinformatics, vol. 34, no. 9, pp. 1615-1617, 2017.

[20] S. J. Turley, V. Cremasco, and J. L. Astarita, "Immunological hallmarks of stromal cells in the tumour microenvironment," Nature Reviews. Immunology, vol. 15, no. 11, pp. 669-682, 2015.

[21] Q. Chen, X. Liu, L. Xu et al., "Long non-coding RNA BACE1AS is a novel target for anisomycin-mediated suppression of ovarian cancer stem cell proliferation and invasion," Oncology Reports, vol. 35, no. 4, pp. 1916-1924, 2016.

[22] Y. Y. Nie, Y. Q. Li, Y. H. Xu, Y. Jiao, and W. Li, "Long noncoding RNA BACE1-AS is an independent unfavorable prognostic factor in liver cancer," Oncology Letters, vol. 20, no. 5, p. 1, 2020.

[23] J. Chen, H. Zhang, L. Zhou et al., "Enhancing the efficacy of tumor vaccines based on immune evasion mechanisms," Frontiers in Oncology, vol. 10, 2021.

[24] J. D. Armitage, H. V. Newnes, A. Mcdonnell, A. Bosco, and J. Waithman, "Fine-tuning the tumour microenvironment: current perspectives on the mechanisms of tumour immunosuppression," Cells, vol. 10, no. 1, p. 56, 2021.

[25] J. Arneth, "Tumor microenvironment," Medicina (Kaunas, Lithuania), vol. 56, no. 1, p. 15, 2019.

[26] C. I. Diakos, K. A. Charles, D. C. Mcmillan, and S. J. Clarke, "Cancer-related inflammation and treatment effectiveness," Lancet Oncology, vol. 15, no. 11, pp. e493-e503, 2014.

[27] J. Candido and T. Hagemann, "Cancer-related inflammation," Journal of Clinical Immunology, vol. 33, Suppl 1, pp. S79-S84, 2013.

[28] X. Wu, M. Peng, B. Huang et al., "Immune microenvironment profiles of tumor immune equilibrium and immune escape states of mouse sarcoma," Cancer Letters, vol. 340, no. 1, pp. 124-133, 2013.

[29] R. V. Sionov, Z. G. Fridlender, and Z. Granot, "The multifaceted roles neutrophils play in the tumor microenvironment," Cancer Microenvironment, vol. 8, no. 3, pp. 125-158, 2015.

[30] A. Cerwenka and L. L. Lanier, "Natural killers join the fight against cancer," Science, vol. 359, no. 6383, pp. 1460-1461, 2018.

[31] A. Schmieder, J. Michel, K. Schonhaar, S. Goerdt, and K. Schledzewski, "Differentiation and gene expression profile of tumor-associated macrophages," Seminars in Cancer Biology, vol. 22, no. 4, pp. 289-297, 2012.

[32] M. M. Kaneda, K. S. Messer, N. Ralainirina et al., "PI3K $\gamma$ is a molecular switch that controls immune suppression," Nature, vol. 539, no. 7629, pp. 437-442, 2016.

[33] D. M. Pardoll, "The blockade of immune checkpoints in cancer immunotherapy," Nature Reviews. Cancer, vol. 12, no. 4, pp. 252-264, 2012.

[34] N. Curdy, O. Lanvin, C. Laurent, J. J. Fournie, and D. M. Franchini, "Regulatory mechanisms of inhibitory immune checkpoint receptors expression," Trends in Cell Biology, vol. 29, no. 10, pp. 777-790, 2019.

[35] I. Murtaza, V. M. Adhami, B. Hafeez, M. Saleem, and H. Mukhtar, "Fisetin, a natural flavonoid, targets chemoresistant human pancreatic cancer AsPC-1 cells through DR3mediated inhibition of NF-kappaB," International Journal of Cancer Journal International Du Cancer, vol. 125, no. 10, pp. 2465-2473, 2010.
[36] C. Cavallini, O. Lovato, A. Bertolaso et al., "Expression and function of the TL1A/DR3 axis in chronic lymphocytic leukemia," Oncotarget, vol. 6, no. 31, pp. 32061-32074, 2015.

[37] C. Z. You, Q. G. Liu, C. Xiao, G. N. Wang, and X. W. Feng, "The role of death receptor 3 in the biological behavior of hepatocellular carcinoma cells," Molecular Medicine Reports, vol. 11, no. 2, pp. 797-804, 2015.

[38] S. Gout, C. Morin, F. Houle, and J. Huot, "Death receptor-3, a new E-selectin counter-receptor that confers migration and survival advantages to colon carcinoma cells by triggering p38 and ERK MAPK activation," Cancer Research, vol. 66, no. 18, pp. 9117-9124, 2006.

[39] Z. Ge, A. J. Sanders, L. Ye, R. E. Mansel, and W. G. Jiang, "Expression of death receptor-3 in human breast cancer and its functional effects on breast cancer cells in vitro," Oncology Reports, vol. 29, no. 4, pp. 1356-1364, 2013. 